\title{
Product of invariant types modulo domination-equivalence
}

\author{
Rosario Mennuni ${ }^{1}$
}

Received: 31 October 2018 / Accepted: 19 April 2019 / Published online: 9 May 2019

(c) The Author(s) 2019

\begin{abstract}
We investigate the interaction between the product of invariant types and dominationequivalence. We present a theory where the latter is not a congruence with respect to the former, provide sufficient conditions for it to be, and study the resulting quotient when it is.
\end{abstract}

Keywords Domination · Domination-equivalence $\cdot$ Equidominance $\cdot$ Product of invariant types

\section{Mathematics Subject Classification 03C45}

To a sufficiently saturated model of a first-order theory one can associate a semigroup, that of global invariant types with the tensor product $\otimes$. This can be endowed with two equivalence relations, called domination-equivalence and equidominance. This paper studies the resulting quotients, starting from sufficient conditions for $\otimes$ to be well-defined on them. We show, correcting a remark in [3], that this need not be always the case.

Let $S(\mathfrak{U})$ be the space of types in any finite number of variables over a model $\mathfrak{U}$ of a first-order theory that is $\kappa$-saturated and $\kappa$-strongly homogeneous for some large $\kappa$. For any set $A \subseteq \mathfrak{U}$, one has a natural action on $S(\mathfrak{U})$ by the group $\operatorname{Aut}(\mathfrak{U} / A)$ of automorphisms of $\mathfrak{U}$ that fix $A$ pointwise. The space $S^{\text {inv }}(\mathfrak{U})$ of global invariant types consists of those elements of $S(\mathfrak{U})$ which, for some small $A$, are fixed points of the action Aut $(\mathfrak{U} / A) \curvearrowright S(\mathfrak{U})$. Each of these types has a canonical extension to bigger models $\mathfrak{U}_{1} \succ \mathfrak{U}$, namely the unique one which is a fixed point of the action $\operatorname{Aut}\left(\mathfrak{U}_{1} / A\right) \curvearrowright S\left(\mathfrak{U}_{1}\right)$, and this allows us to define an associative product $\otimes$ on $S^{\text {inv }}(\mathfrak{U})$. This is the semigroup which we are going to quotient.

We say that a global type $p$ dominates a global type $q$ when $p$ together with a small set of formulas entails $q$. This is a preorder, and we call the induced equivalence relation domination-equivalence. We also look at equidominance, the refinement of

\footnotetext{
$\bowtie$ Rosario Mennuni

R.Mennuni@leeds.ac.uk

1 University of Leeds, Leeds, UK
} 
domination-equivalence obtained by requiring that domination of $p$ by $q$ and of $q$ by $p$ can be witnessed by the same set of formulas. These notions have their roots in the work of Lascar, who in [9] generalised the Rudin-Keisler order on ultrafilters to types of a theory; his preorder was subsequently generalised to domination between stationary types in a stable theory.

Equidominance reached its current form in [3], where it was used to prove a result of Ax-Kochen-Ershov flavour; namely, that in the case of algebraically closed valued fields one can compute the quotient of the semigroup of global invariant types by equidominance, and it turns out to be commutative and to decompose in terms of value group and residue field. It was also claimed, without proof, that such a semigroup is also well-defined and commutative in any complete first-order theory. The starting point of this research was to try to fill this gap by proving these claims. After trying in vain to prove well-definedness of the quotient semigroup, the author started to investigate sufficient conditions for it to hold. Eventually, a counterexample arose:

Theorem There is a ternary, $\omega$-categorical, supersimple theory of SU-rank 2 with degenerate algebraic closure in which neither domination-equivalence nor equidominance are congruences with respect to $\otimes$.

The paper is organised as follows. In Sect. 1 we define the main object of study, namely the quotient $\widetilde{\operatorname{Inv}}(\mathfrak{U})$ of the semigroup of global invariant types modulo domination-equivalence, provide some sufficient conditions for it to be well-defined and investigate its most basic properties. In Sect. 2 we prove the theorem above, which shows that $\widetilde{\operatorname{Inv}}(\mathfrak{U})$ need not be well-defined in general; we also show (Corollary 2.12) that in the theory of the Random Graph $\widetilde{\operatorname{Inv}}(\mathfrak{U})$ is not commutative. In Sect. 3 we prove that definability, finite satisfiability, generic stability (Theorem 3.5) and weak orthogonality to a type (Proposition 3.13) are preserved downwards by domination. This is useful in explicit computations of Inv $(\mathfrak{U})$ and yields as a by-product (Corollary 3.11) that another, smaller object based on generically stable types is instead well-defined in full generality. In Sect. 4 we explore whether and how much $\widetilde{\operatorname{Inv}}(\mathfrak{U})$ depends on $\mathfrak{U}$; we show (Corollary 4.7) that its independence from the choice of $\mathfrak{U}$ implies NIP. Section 5 gathers some previously known results from classical stability theory and explores their consequences in the context of this paper (e.g. Theorem 5.11). Sections from 2 to 4 depend on Sect. 1 but can be read independently of each other; Sect. 5 contains references to all previous sections but can in principle be read after Sect. 1.

\section{Definition and well-definedness}

\subsection{Set-up}

Notations and conventions are standard, and we now recall some of them.

We work in an arbitrary complete theory $T$, in a first-order language $L$, with infinite models. As customary, all mentioned inclusions between models of $T$ are assumed to be elementary maps, and we call models of $T$ which are $\kappa$-saturated and $\kappa$-strongly homogeneous for a large enough $\kappa$ "monster" models; we denote them by $\mathfrak{U}, \mathfrak{U}_{0}$, etc. Saying that $A \subseteq \mathfrak{U}$ is small means that $\mathfrak{U}$ is $|A|^{+}$-saturated and $|A|^{+}$-strongly 
homogeneous, and is sometimes denoted by $A \subset^{+} \mathfrak{U}$, or $A \prec^{+} \mathfrak{U}$ if additionally $A \prec \mathfrak{U}$. Large means "not small". The letters $A$ and $M$ usually represent, respectively, a small subset and a small elementary substructure of $\mathfrak{U}$.

Parameters and variables are tacitly allowed to be finite tuples unless otherwise specified, and we abuse the notation by writing e.g. $a \in \mathfrak{U}$ instead of $a \in \mathfrak{U}^{|a|}$. Coordinates of a tuple are indicated with subscripts, starting with 0 , so for instance $a=\left(a_{0}, \ldots, a_{|a|-1}\right)$. To avoid confusion, indices for a sequence of tuples are written as superscripts, as in $\left\langle a^{i} \mid i \in I\right\rangle$. The letters $x, y, z, w, t$ denote tuples of variables, the letters $a, b, c, d, e, m$ denote tuples of elements of a model.

A global type is a complete type over $\mathfrak{U}$. "Type over $B$ " means "complete type over $B$ ". We say "partial type" otherwise. We sometimes write e.g. $p_{x}$ in place of $p(x)$ and denote with $S_{x}(B)$ the space of types in variables $x$.

When mentioning realisations of global types, or supersets of a monster, we implicitly think of them as living inside a bigger monster model, which usually goes unnamed. Similarly, implications are to be understood modulo the elementary diagram $\operatorname{ED}\left(\mathfrak{U}_{*}\right)$ of an ambient monster model $\mathfrak{U}_{*}$, e.g. if $c \in \mathfrak{U}_{*} \succ \mathfrak{U}$ and $p \in S(\mathfrak{U} c)$ then $(p\lceil\mathfrak{U}) \vdash p$ is a shorthand for $(p \nmid \mathfrak{U}) \cup \operatorname{ED}\left(\mathfrak{U}_{*}\right) \vdash p$. We sometimes take deductive closures implicitly, as in " $\{x=a\} \in S_{x}(\mathfrak{U})$ ".

If we define a property a theory may have, and then we say that a structure has it, we mean that its complete theory does. When we say " $L$-formula", we mean without parameters; for emphasis, we sometimes write $L(\emptyset)$, with the same meaning as $L$. In formulas, (tuples of) variables will be separated by commas or semicolons. The distinction is purely cosmetic, to help readability, and usually it means we regard the variables on the left of the semicolon as "object variables" and the ones on the right as "parameter variables", e.g. we may write $\varphi(x, y ; w) \in L, \varphi(x, y ; d) \in p(x) \otimes q(y)$.

\subsubsection{Products of invariant types}

We briefly recall some standard results on invariant types and fix some notation. For proofs, see e.g. [15, Section 2.2] or [13, Chapter 12].

Definition 1.1 Let $A \subseteq B$. A type $p \in S_{x}(B)$ is $A$-invariant iff for all $\varphi(x ; y) \in L$ and $a \equiv_{A} b$ in $B^{|y|}$ we have $p(x) \vdash \varphi(x ; a) \leftrightarrow \varphi(x ; b)$. A global type $p \in S_{x}(\mathfrak{U})$ is invariant iff it is $A$-invariant for some small $A$.

Equivalently, a global $p \in S_{x}(\mathfrak{U})$ is $A$-invariant iff it is a fixed point of the usual action of Aut $(\mathfrak{U} / A)$ on $S_{x}(\mathfrak{U})$ defined by $f(p):=\{\varphi(x ; f(a)) \mid \varphi(x ; y) \in L(\emptyset), \varphi(x ; a) \in$ $p$ \}. Note that if $p$ is $A$-invariant and $A_{1} \supseteq A$, then $p$ is automatically $A_{1}$-invariant. This will be used tacitly throughout.

Notation We denote by $S_{x}^{\text {inv }}(\mathfrak{U}, A)$ the space of global $A$-invariant types in variables $x$, with $A$ small, and with $S_{x}^{\text {inv }}(\mathfrak{U})$ the union of the $S_{x}^{\text {inv }}(\mathfrak{U}, A)$ as $A$ varies among small subsets of $\mathfrak{U}$. We denote by $S_{<\omega}(B)$, or just by $S(B)$, the union for $n<\omega$ of the spaces of complete types in $n$ variables over $B$. Similarly for, say, $S_{<\omega}^{\text {inv }}(\mathfrak{U})$.

If $p \in S_{x}(\mathfrak{U})$ is $A$-invariant and $\varphi(x ; y) \in L$, write

$$
\left(d_{p} \varphi(x ; y)\right)(y):=\left\{\operatorname{tp}_{y}(b / A) \mid \varphi(x ; b) \in p, b \in \mathfrak{U}\right\}
$$


If $p(x), q(y) \in S(B)$ and $A \subseteq B$, we write

$$
S_{p q}(A):=\left\{r \in S_{x y}(A) \mid r \supseteq(p\lceil A) \cup(q \uparrow A)\}\right.
$$

In situations like the one above, we implicitly assume, for convenience and with no loss of generality, that $x$ and $y$ share no common variable.

Proposition 1.2 [15, p. 19] Let A be small. Given an A-invariant type $p \in S_{x}(\mathfrak{U})$ and a set of parameters $B \supseteq \mathfrak{U}$ there is a unique extension $p \mid B$ of $p$ to an A-invariant type over $B$, and it is given by requiring, for all $\varphi(x ; y) \in L$ and $b \in B$,

$$
\varphi(x ; b) \in p \mid B \Longleftrightarrow \operatorname{tp}(b / A) \in\left(d_{p} \varphi(x ; y)\right)(y)
$$

Moreover, if $p \in S_{x}^{\operatorname{inv}}(\mathfrak{U}, A), \varphi(x, y ; w) \in L(\emptyset), d \in \mathfrak{U}$ and $q \in S_{y}(\mathfrak{U})$, then the following are equivalent:

1. For some (equivalently, all) $b \vDash q$ we have that $\varphi(x, b ; d) \in p \mid \mathfrak{U} b$.

2. For some (equivalently, all) $b \in \mathfrak{U}$ such that $b \vDash q\lceil A d$ we have that $\varphi(x, b ; d) \in$ $p$.

3. $q \in \pi^{-1}\left(\left(d_{p} \varphi(x, y ; d)\right)(y)\right)$, for $\pi: S_{y}(\mathfrak{U}) \rightarrow S_{y}(A d)$ the restriction map.

Also note that if $A_{1} \supseteq A$ is another small set then $p \mid B$ is also the unique $A_{1}$-invariant extension of $p$. All this ensures that the following operation is well-defined, i.e. does not depend on $b \vDash q$ and on whether we regard $p$ as $A$-invariant or $A_{1}$-invariant.

Definition 1.3 Let $p \in S_{x}^{\text {inv }}(\mathfrak{U}, A)$ and $q \in S_{y}(\mathfrak{U})$. Define $p(x) \otimes q(y) \in S_{x y}(\mathfrak{U})$ as follows. Fix $b \vDash q$. For each $\varphi(x, y) \in L(\mathfrak{U})$, define

$$
\varphi(x, y) \in p(x) \otimes q(y) \Longleftrightarrow \varphi(x, b) \in p \mid \mathfrak{U} b
$$

We also define inductively $p^{(1)}:=p\left(x^{0}\right)$ and $p^{(n+1)}:=p\left(x^{n}\right) \otimes p^{(n)}\left(x^{n-1}, \ldots, x^{0}\right)$.

Fact 1.4 [15, Fact 2.19 and Fact 2.20] The product of two $A$-invariant global types is still $A$-invariant, and $\otimes$ is associative on $S^{\text {inv }}(\mathfrak{U})$.

Example 1.5 If $T$ is stable then $S^{\text {inv }}(\mathfrak{U})=S(\mathfrak{U})$ and $p \otimes q=\operatorname{tp}(a, b / \mathfrak{U})$ where $a \vDash p$, $b \vDash q$ and $a \bigsqcup_{\mathfrak{U}} b$. If $T=\mathrm{DLO}$ and $p(x)=\operatorname{tp}(+\infty / \mathfrak{U})$, then $p(x) \otimes p(y)=$ $p(x) \cup p(y) \cup\{x>y\}$.

\subsubsection{Domination}

Definition 1.6 Let $p \in S_{x}(\mathfrak{U})$ and $q \in S_{y}(\mathfrak{U})$.

1. We say that $p$ dominates $q$ and write $p \geq_{\mathrm{D}} q$ iff there are some small $A$ and some $r \in S_{x y}(A)$ such that

- $r \in S_{p q}(A)$, and

- $p(x) \cup r(x, y) \vdash q(y)$. 
2. We say that $p$ and $q$ are domination-equivalent and write $p \sim_{\mathrm{D}} q$ iff $p \geq_{\mathrm{D}} q$ and $q \geq \mathrm{D} p$.

3. We say that $p$ and $q$ are equidominant and write $p \equiv_{\mathrm{D}} q$ iff there are some small $A$ and some $r \in S_{x y}(A)$ such that

- $r \in S_{p q}(A)$,

- $p(x) \cup r(x, y) \vdash q(y)$, and

- $q(y) \cup r(x, y) \vdash p(x)$.

So $p \equiv_{\mathrm{D}} q$ if and only if both $p \geq_{\mathrm{D}} q$ and $q \geq_{\mathrm{D}} p$ hold, and both statements can be witnessed by the same $r$. To put it differently, a direct definition of $p \sim_{\mathrm{D}} q$ can be obtained by replacing, in the last clause of the definition of $p \equiv_{\mathrm{D}} q$, the small type $r$ with another small type $r^{\prime}$, possibly different from $r$. That the last two relations are in general distinct can be seen for instance in DLO together with a dense-codense predicate; see Example 1.11.

Note that we are not requiring $p \cup r$ to be complete; in other words, domination is "small-type semi-isolation", as opposed to "small-type isolation". The finer relation of semi-isolation, also known as the global RK-order, ${ }^{1}$ was studied for instance in [16].

Proposition $1.7 \geq_{\mathrm{D}}$ and $\equiv_{\mathrm{D}}$ are respectively a preorder and an equivalence relation on $S_{<\omega}(\mathfrak{U})$. Consequently, $\sim_{\mathrm{D}}$ is an equivalence relation as well.

Proof The only non-obvious thing is transitivity. We prove it for $\equiv_{\mathrm{D}}$ first, as the proof for $\geq_{\mathrm{D}}$ is even easier. Suppose that $r(x, y) \in S_{p_{0} p_{1}}\left(A_{r}\right)$ witnesses that $p_{0}(x) \equiv_{\mathrm{D}}$ $p_{1}(y)$ and that $s(y, z) \in S_{p_{1} p_{2}}\left(A_{s}\right)$ witnesses $p_{1}(y) \equiv_{\mathrm{D}} p_{2}(z)$. Up to taking a larger $A$ and then completing $r, s$ to types with parameters from $A$, we can assume $A_{r}=$ $A_{s}=A$. By hypothesis and compactness, for every formula $\varphi(z) \in p_{2}$ there are formulas $\psi(y, z) \in s, \theta(y) \in p_{1}$ and $\chi(x, y) \in r$ such that $p_{0} \cup\{\chi(x, y)\} \vdash \theta(y)$ and $\{\theta(y) \wedge \psi(y, z)\} \vdash \varphi(z)$. If we let $\sigma_{\varphi}(x, z):=\exists y(\chi(x, y) \wedge \psi(y, z))$, then $p_{0}(x) \cup\left\{\sigma_{\varphi}(x, z)\right\} \vdash \varphi(z)$. Moreover, we have $\sigma_{\varphi}(x, z) \in L(A)$. Analogously, for each $\delta(x) \in p_{0}$ we can find $\rho_{\delta}(z, x) \in L(A)$ such that $p_{2}(z) \cup\left\{\rho_{\delta}(z, x)\right\} \vdash \delta(x)$, obtained in the same way mutatis mutandis. It is now enough to show that the set

$$
\Phi:=p_{0}(x) \cup r(x, y) \cup p_{1}(y) \cup s(y, z) \cup p_{2}(z)
$$

is consistent, as this will in particular entail consistency of

$$
\left\{\sigma_{\varphi} \mid \varphi \in p_{2}\right\} \cup\left\{\rho_{\delta} \mid \delta \in p_{0}\right\} \cup\left(p _ { 0 } \lceil A ) \cup \left(p_{2}\lceil A)\right.\right.
$$

which will therefore have a completion to a type in $S_{p_{0} p_{2}}(A)$ witnessing $p_{0} \equiv_{\mathrm{D}} p_{2}$. To see that $\Phi$ is consistent, in a larger monster $\mathfrak{U}_{1}$ let $(a, b) \vDash p_{0} \cup r$ and $(\tilde{b}, \tilde{c}) \vDash p_{1} \cup s$. Since $\operatorname{tp}(b / \mathfrak{U})=p_{1}=\operatorname{tp}(\tilde{b} / \mathfrak{U})$, there is $f \in \operatorname{Aut}\left(\mathfrak{U}_{1} / \mathfrak{U}\right)$ such that $f(\tilde{b})=b$, and then $(a, b, f(\tilde{c})) \vDash \Phi$.

The proof for $\geq_{\mathrm{D}}$ is exactly the same, except we do not need to consider the $\rho_{\delta}$ formulas.

\footnotetext{
1 Strictly speaking, the original definition of the RK-order in [9, Définition 1] slightly differs from the relation that customarily bears the same name in the literature.
} 
As we are interested in the interaction of these notions with $\otimes$, we restrict our attention to quotients of $S^{\text {inv }}(\mathfrak{U})$. Note that, by the following lemma, whether or not $p \in S^{\text {inv }}(\mathfrak{U})$ only depends on its equivalence class.

Lemma 1.8 If $p \in S_{x}^{\mathrm{inv}}(\mathfrak{U}, A)$ and $r \in S_{x y}(B)$ are such that $p \cup r$ is consistent and $p \cup r \vdash q \in S_{y}(\mathfrak{U})$, then $q$ is invariant over $A B$.

Proof The set of formulas $p \cup r$ is fixed by Aut $(\mathfrak{U} / A B)$ and implies $q$. As $q$ is complete, the conclusion follows.

Anyway, $q$ will not be in general $A$-invariant: for instance, by the proof of point 3 of Proposition 1.19 , for every $p$ and every realised $q$ we have $p \geq_{\mathrm{D}} q$, and it is enough to take $q$ realised in $\mathfrak{U} \backslash \operatorname{dcl}(A)$ to get a counterexample.

Definition 1.9 Let $\widetilde{\operatorname{Inv}}(\mathfrak{U})$ be the quotient of $S^{\operatorname{inv}}(\mathfrak{U})$ by $\sim_{D}$, and $\overline{\operatorname{Inv}}(\mathfrak{U})$ the quotient of $S^{\text {inv }}(\mathfrak{U})$ by $\equiv_{\mathrm{D}}$.

Note that, if $p \cup r \vdash q$, by passing to a suitable extension of $r$ there is no harm in enlarging its domain, provided it stays small. This sort of manipulation will from now on be done tacitly.

Remark 1.10 In [3], the name domination-equivalence is used to refer to $\equiv_{\mathrm{D}}$ (no mention is made of $\geq_{D}$ and $\sim_{D}$ ). The reason for this change in terminology is to ensure consistency with the notions with the same names classically defined for stable theories, which coincide with the ones just defined (see Sect. 5). As $\widetilde{\operatorname{Inv}}(\mathfrak{U})$ carries a poset structure, and is in some sense better behaved than $\overline{\operatorname{Inv}}(\mathfrak{U})$, we mostly focus on the former.

Example 1.11 1. It is easy to see that, in any strongly minimal theory, two global types are domination-equivalent, equivalently equidominant, precisely when they have the same dimension over $\mathfrak{U}$.

2. In DLO, if $p(x)$ is the type at $+\infty$, then $p(x) \equiv_{\mathrm{D}} p(y) \otimes p(z)$, as can be easily seen by using some $r$ containing the formula $x=z$.

3. The two equivalence relations differ in the theory DLOP of a DLO with a densecodense predicate $P$. In this case, if $p(x)$ is the type at $+\infty$ in $P$, and $q(y)$ is the type at $+\infty$ in $\neg P$, then $p(x) \geq_{\mathrm{D}} q(y)$ (resp. $\left.p(x) \leq_{\mathrm{D}} q(y)\right)$ can be witnessed by any $r$ containing $y>x$ (resp. $y<x$ ). To show $p \neq_{\mathrm{D}} q$, take any $r \in S_{p q}(A)$. Since $(p(x)\lceil\emptyset) \cup(q(y) \uparrow \emptyset) \vdash P(x) \wedge \neg P(y)$ we have $r \vdash x \neq y$, and since $A$ is small there is $b \in \mathfrak{U}$ such that $b>A$. It follows from quantifier elimination that, if for instance $r \vdash x>y$, then $p \cup r \forall y>b$, and a fortiori $p \cup r \forall q$. The reason the two equivalence relations may differ is, simply, that even if there are $r_{0}$ and $r_{1}$ such that $p \cup r_{0} \vdash q$ and $q \cup r_{1} \vdash p$, we may still have that the union $r_{0} \cup r_{1}$ is inconsistent.

4. The two equivalence relations may differ even in a stable theory, as shown by [17, Example 5.2.9] together with the fact (Proposition 5.4) that the classical definitions via forking (see Definition 5.3) in stable theories coincide with the ones in Definition 1.6. 


\subsubsection{Interaction with $\otimes$}

We start our investigation of the compatibility of $\otimes$ with $\geq_{D}$ and $\equiv_{D}$ with two easy lemmas. While the first one will not be needed until later, the second one will be used repeatedly.

Lemma 1.12 If $A \subseteq B \subseteq C, p_{x}, q_{y} \in S(C)$ and $r \in S_{p q}(A)$ is such that $p \cup r \vdash q$, then $(p\lceil B) \cup r \vdash q \uparrow B$.

Proof Let $\psi(y) \in q\lceil B$. By hypothesis and compactness there is $\chi(x, y) \in r$ such that $p \vdash \forall y(\chi(x, y) \rightarrow \psi(y))$. As $A \subseteq B$, this formula is in $p\lceil B$.

Lemma 1.13 If $p_{x}, q_{y} \in S^{\mathrm{inv}}(\mathfrak{U}, A)$ and $r \in S_{p q}(A)$ is such that $p \cup r \vdash q$, then for all sets of parameters $B \supseteq \mathfrak{U}$ we have $(p \mid B) \cup r \vdash q \mid B$.

Proof Let $\varphi(y ; w)$ be an $L(\emptyset)$-formula and $b \in B$ be such that $\varphi(y ; b) \in q \mid B$. Pick any $\tilde{b} \in \mathfrak{U}$ such that $\tilde{b} \equiv_{A} b$. By definition of $q \mid B$ we have $\varphi(y ; \tilde{b}) \in q$, so by hypothesis and compactness there is an $L(A)$-formula $\psi(x, y) \in r(x, y)$ such that $p \vdash \forall y(\psi(x, y) \rightarrow \varphi(y ; \tilde{b}))$. But then, by definition of $p \mid B$ and the fact that $\psi \in L(A)$ we have $p \mid B \vdash \forall y(\psi(x, y) \rightarrow \varphi(y ; b))$, and since $\psi \in r$ we get $(p \mid B) \cup r \vdash \varphi(y ; b)$.

Notation We adopt from now on the following conventions. The letter $A$ continues to denote a small set. The symbols $p, q$, possibly with subscripts, denote global $A$ invariant types, and $r$ stands for an element of, say, $S_{p q}(A)$ witnessing domination or equidominance.

The first use we make of Lemma 1.13 is to prove the following statement, which generalises [9, Corollaire 11].

Lemma 1.14 Suppose $p_{0}(x) \cup r(x, y) \vdash p_{1}(y)$, and let $s:=r(x, y) \cup\{z=w\}$. Then $\left(p_{0}(x) \otimes q(z)\right) \cup s \vdash p_{1}(y) \otimes q(w)$. In particular if $p_{0} \geq_{\mathrm{D}} p_{1}$ then $p_{0} \otimes q \geq_{\mathrm{D}} p_{1} \otimes q$, and the same holds replacing $\geq_{\mathrm{D}}$ with $\equiv_{\mathrm{D}}$.

Proof Choose any $b \vDash q(z)$. For any $\varphi(y, z ; t) \in L(\emptyset)$ and $d \in \mathfrak{U}$ such that $\varphi(y, z ; d) \in p_{1}(y) \otimes q(z)$ we have, by definition of $\otimes$, that $p_{1}(y) \mid \mathfrak{U} b \vDash \varphi(y, b ; d)$. By Lemma 1.13 there is some $L(A)$-formula $\psi(x, y) \in r(x, y)$ such that $p_{0}(x) \mid \mathfrak{U} b \vDash$ $\forall y(\psi(x, y) \rightarrow \varphi(y, b ; d))$, hence $p_{0}(x) \otimes q(z) \vDash \forall y(\psi(x, y) \rightarrow \varphi(y, z ; d))$. In particular, since $\psi \in r$, we have $\left(p_{0}(x) \otimes q(z)\right) \cup r \vdash \varphi(y, z ; d)$. Therefore any completion of $s \cup\left(\left(p_{0}(x) \otimes q(z)\right) \uparrow A\right) \cup\left(\left(p_{1}(y) \otimes q(w)\right) \uparrow A\right)$ witnesses that $p_{0}(x) \otimes q(z) \geq_{\mathrm{D}} p_{1}(y) \otimes q(w)$.

In the special case where the same $r$ also witnesses $p_{1} \geq_{\mathrm{D}} p_{0}$, for the same $s$ we have that $s \cup\left(\left(p_{0}(x) \otimes q(z)\right) \uparrow A\right) \cup\left(\left(p_{1}(y) \otimes q(w)\right) \uparrow A\right)$ witnesses $p_{1} \otimes q \geq_{\mathrm{D}} p_{0} \otimes q$, and we get $p_{1} \otimes q \equiv_{\mathrm{D}} p_{0} \otimes q$.

One may expect a similar result to hold when multiplying on the left by $p$ a relation of the form $q_{0} \geq_{\mathrm{D}} q_{1}$, and indeed it was claimed (without proof) in [3] that $\equiv_{\mathrm{D}}$ is a congruence with respect to $\otimes$. Unfortunately, this turns out not be true in general: we will see in Sect. 2 that it is possible to have $q_{0} \equiv_{\mathrm{D}} q_{1}$ and $p \otimes q_{0} \Varangle_{\mathrm{D}} p \otimes q_{1}$ simultaneously. For the time being, we assume this does not happen as an hypothesis and explore some of its immediate consequences. 
Definition 1.15 For a theory $T$, we say that $\otimes$ respects (or is compatible with) $\geq_{\mathrm{D}}$ (resp. $\equiv_{\mathrm{D}}$ ) iff for all global invariant types $p, q_{0}, q_{1}$, if $q_{0} \geq_{\mathrm{D}} q_{1}\left(\right.$ resp. $q_{0} \equiv_{\mathrm{D}} q_{1}$ ) then $p \otimes q_{0} \geq_{\mathrm{D}} p \otimes q_{1}$ (resp. $p \otimes q_{0} \equiv_{\mathrm{D}} p \otimes q_{1}$ ).

Corollary 1.161 . $\otimes$ respects $\geq_{\mathrm{D}}$ if and only if $\left(S^{\mathrm{inv}}(\mathfrak{U}), \otimes, \geq_{\mathrm{D}}\right)$ is a preordered semigroup. In this case $\sim_{\mathrm{D}}$ is a congruence with respect to $\otimes$, and the latter induces on $\left(\widetilde{\operatorname{Inv}}(\mathfrak{U}), \geq_{\mathrm{D}}\right)$ the structure of a partially ordered semigroup.

2. $\otimes$ respects $\equiv_{\mathrm{D}}$ if and only if $\equiv_{\mathrm{D}}$ is a congruence with respect to $\otimes$.

Proof Everything follows at once from Lemma 1.14.

Lemma 1.17 Suppose that $p, q \in S^{\text {inv }}(\mathfrak{U})$ and $p$ is realised. The following are equivalent: 1. $p \equiv_{\mathrm{D}}$ q. 2. $p \sim_{\mathrm{D}}$ q. 3. $p \geq_{\mathrm{D}}$ q. 4. $q$ is realised.

Proof The implications $1 \Rightarrow 2 \Rightarrow 3$ are true by definition, even when $p$ is not realised. Let $p=\operatorname{tp}(a / \mathfrak{U})$, where $a \in \mathfrak{U}$.

For $3 \Rightarrow 4$ suppose that $r \in S_{p q}(A)$ is such that $p \cup r \vdash q$. Since $\{x=a\} \vdash p$, we have $\{x=a\} \cup r \vdash q$. But since $\{x=a\} \cup r$ is a small type, it is realised in $\mathcal{U}$ by some $(a, b)$, and clearly $b \vDash q$.

For $4 \Rightarrow 1$ suppose that for some $b \in \mathfrak{U}$ we have $q=\operatorname{tp}(b / \mathfrak{U})$ and let $A$ be any small set containing $a$ and $b$. Clearly, $(x=a) \wedge(y=b)$ implies a complete type $r \in S_{x y}(A)$ containing $(p\lceil A) \cup(q\lceil A)$, and since $r(x, y) \vdash p(x) \cup q(y)$ we have that $r$ witnesses $p \equiv_{\mathrm{D}} q$.

Lemma 1.18 Suppose that $p_{x}, q_{y} \in S^{\mathrm{inv}}(\mathfrak{U})$ and that $p$ is realised by $a \in \mathfrak{U}$. Then $\{x=a\} \cup q(y) \vdash p(x) \cup q(y) \vdash p(x) \otimes q(y)=q(y) \otimes p(x)$. Moreover, $p(x) \otimes$ $q(y) \equiv_{\mathrm{D}} q(y)$.

Proof The first part is clear. It follows that, if $q$ is $A$-invariant and $a \in A$, in order to show that $p(x) \otimes q(y) \equiv_{\mathrm{D}} q(z)$ it suffices to take as $r$ the type $\{x=a\} \cup\{y=$ z\} $\cup(q(y)\lceil A) \cup(q(z)\lceil A)$.

Notation When quotienting by $\sim_{\mathrm{D}}$ or $\equiv_{\mathrm{D}}$ we denote by $\llbracket p \rrbracket$ the class of $p$, with the understanding that the equivalence relation we are referring to is clear from context. We write $\llbracket 0 \rrbracket$ for the class of realised types. ${ }^{2}$

Proposition 1.19 Suppose that $\otimes$ respects $\geq_{\mathrm{D}}\left(\right.$ resp. $\left.\equiv_{\mathrm{D}}\right)$. Then:

1. $(\widetilde{\operatorname{Inv}}(\mathfrak{U}), \otimes)($ resp. $(\overline{\operatorname{Inv}}(\mathfrak{U}), \otimes))$ has neutral element $\llbracket 0 \rrbracket$;

2. no element different from $\llbracket 0 \rrbracket$ is invertible;

3. in $\widetilde{\operatorname{Inv}}(\mathfrak{U})$, $\llbracket 0 \rrbracket$ is the minimum of $\geq \mathrm{D}$.

Proof 1. Let $p=\operatorname{tp}(a / \mathfrak{U})$ and $q \in S^{\text {inv }}(\mathfrak{U})$, where $a \in \mathfrak{U}$. Apply Lemma 1.18 and note that $p \otimes q \equiv_{\mathrm{D}} q$ implies $p \otimes q \sim_{\mathrm{D}} q$.

2. By the previous point if $\llbracket p \rrbracket$ is invertible then there is some $q \in S^{\text {inv }}(\mathfrak{U})$ such that $p \otimes q$ is realised. In particular, $p$ is realised as well.

3. We have to show that for every $p(x)$ and every realised $q(y)$ we have $p \geq_{\mathrm{D}} q$. If $q$ is realised by $b \in \mathfrak{U}$, it is sufficient to put in $r$ the formula $y=b$.

2 It is the class of the unique global 0-type. 


\subsection{Some sufficient conditions}

We proceed to investigate sufficient conditions for $\otimes$ to respect $\geq_{\mathrm{D}}$ and $\equiv_{\mathrm{D}}$. These conditions are admittedly rather artificial, but we show they are a consequence of other properties that are easier to test directly, such as stability.

In what follows, types will be usually assumed to have no realised coordinates and no duplicate coordinates, i.e. we will assume, for all $i<j<|x|$ and $a \in \mathfrak{U}$, to have $p(x) \vdash\left(x_{i} \neq a\right) \wedge\left(x_{i} \neq x_{j}\right)$. Up to domination-equivalence, and even equidominance, no generality is lost, as justified by Lemma 1.18 and by the fact that, for example, if $p\left(x_{0}\right)$ is any 1-type and $q\left(y_{0}, y_{1}\right) \vdash p\left(y_{0}\right) \cup\left\{y_{0}=y_{1}\right\}$, setting $x_{0}=y_{0}=y_{1}$ shows $p \equiv_{\mathrm{D}} q$.

We usually abuse the notation and indicate e.g. ( $\left.\widetilde{\operatorname{Inv}}(\mathfrak{U}), \otimes, \leq_{D}\right)$ simply with $\widetilde{\operatorname{Inv}}(\mathfrak{U})$.

Definition 1.20 Let $p, q_{0}, q_{1} \in S^{\text {inv }}(\mathfrak{U})$ and $r \in S_{q_{0} q_{1}}(A)$. Let $\mathfrak{U}_{1}{ }^{+} \succ \mathfrak{U}$ and $b, c \in \mathfrak{U}_{1}$ be such that $(b, c) \vDash q_{0} \cup r \cup q_{1}$, and let $a \vDash p(x) \mid \mathfrak{U}_{1}$. Define

$$
r[p]:=\left(\operatorname{tp}_{x y z}(a b c / A) \cup\{x=w\}\right) \in S_{x y z w}(A)
$$

We say that $T$ has stationary domination (resp. stationary equidominance) iff whenever $p, q_{0}, q_{1} \in S^{\text {inv }}(\mathfrak{U})$ and $q_{0} \geq_{\mathrm{D}} q_{1}$ (resp. $q_{0} \equiv_{\mathrm{D}} q_{1}$ ), there are $A \subset^{+} \mathfrak{U}$ and $r \in S_{q_{0} q_{1}}(A)$ such that

- $p(x), q_{0}(y), q_{1}(z)$ are $A$-invariant,

- $q_{0} \cup r \vdash q_{1}$ (resp. $q_{0} \cup r \vdash q_{1}$ and $q_{1} \cup r \vdash q_{0}$ ), and

- for all $\mathfrak{U}_{1}{ }^{+} \succ \mathfrak{U}$, all $b, c \in \mathfrak{U}_{1}$ such that $(b, c) \vDash q_{0} \cup r$ and all $a \vDash p(x) \mid \mathfrak{U}_{1}$, we have

$$
\left(p(x) \otimes q_{0}(y)\right) \cup r[p] \vdash p(w) \otimes q_{1}(z)
$$

$\left(\right.$ resp. $\left(p(x) \otimes q_{0}(y)\right) \cup r[p] \vdash p(w) \otimes q_{1}(z)$ and $\left(p(w) \otimes q_{1}(z)\right) \cup r[p] \vdash$ $\left.p(x) \otimes q_{0}(y)\right)$.

Proposition 1.21 If $T$ has stationary domination, then $\otimes$ respects $\geq_{\mathrm{D}}$. If $T$ has stationary equidominance, then $\otimes$ respects $\equiv_{\mathrm{D}}$.

Proof Immediate from the definitions.

Definition 1.22 We say that $q_{1}$ is algebraic over $q_{0}$ iff there are $b \vDash q_{0}$ and $c \vDash q_{1}$ such that $c \in \operatorname{acl}(\mathfrak{U} b)$. We say that $T$ has algebraic domination iff $p \geq_{\mathrm{D}} q$ if and only if $q$ is algebraic over $p$.

Proposition 1.23 Suppose that $q_{1}$ is algebraic over $q_{0}$. Then for all $p \in S^{\text {inv }}(\mathfrak{U})$ we have $p \otimes q_{0} \geq_{\mathrm{D}} p \otimes q_{1}$, and this is witnessed by a type $r[p]$ as in the definition of stationary domination. In particular, algebraic domination implies stationary domination.

Proof Let $b, c \in \mathfrak{U}_{1}{ }^{+} \succ \mathfrak{U}$ witness algebraicity of $q_{1}$ over $q_{0}$. Suppose $\psi(y, z)$ is an $L(\mathfrak{U})$-formula such that $\psi(b, z)$ isolates $\operatorname{tp}(c / \mathfrak{U} b)$, and let $s:=\{x=w\} \cup\{\psi(y, z)\}$. 
If $A$ is such that $p \in S^{\text {inv }}(\mathfrak{U}, A)$ and $\psi(y, z) \in L(A)$, let $r:=q_{0}(y)\lceil A \cup\{\psi(y, z)\}$. Then $s \subseteq r[p]$, so it is enough to show that $p(x) \otimes q_{0}(y) \cup s \vdash p(w) \otimes q_{1}(z)$. In some $\mathfrak{U}_{2}{ }^{+} \succ \mathfrak{U}_{1}$, let $a \vDash p \mid \mathfrak{U}_{1}$ and let $\varphi(w, z) \in p(w) \otimes q_{1}(z)$. This means that $\varphi(w, z) \in L(\mathfrak{U})$ and $\varphi(w, c) \in \operatorname{tp}(a / \mathfrak{U} c)=p \mid \mathfrak{U} c$.

By hypothesis, there are only finitely many $\tilde{c} \equiv \mathfrak{U} b c$, which must be contained in any model containing $\mathfrak{U} b$ and, by invariance of $p \mid \mathfrak{U}_{1}$, for all such $\tilde{c} \in \mathfrak{U}_{1}$ we have $p \mid \mathfrak{U}_{1} \vdash \varphi(x, \tilde{c})$. It follows that $\operatorname{tp}\left(a / \mathfrak{U}_{2}\right) \vdash \forall z(\psi(b, z) \rightarrow \varphi(w, z))$. As the latter is an $L(\mathfrak{U} b)$-formula, it is contained in $p \mid \mathfrak{U} b$, and it follows that $\left(p(x) \otimes q_{0}(y)\right) \cup s \vdash$ $p(w) \otimes q_{1}(z)$.

Corollary 1.24 Suppose that $q_{1}$ is the pushforward $f_{*}\left(q_{0}\right)$ of $q_{0}$, for some definable function $f$. Then, for all $p \in S^{\mathrm{inv}}(\mathfrak{U})$, we have $p \otimes q_{0} \geq_{\mathrm{D}} p \otimes q_{1}$.

Proposition 1.25 Let $T$ be stable. Then $T$ has stationary domination and stationary equidominance. Moreover $\widetilde{\operatorname{Inv}}(\mathfrak{U})$ and $\overline{\operatorname{Inv}}(\mathfrak{U})$ are commutative.

Proof Let $r$ witness $q_{0}(y) \geq_{\mathrm{D}} q_{1}(z)$. By Lemma $1.14\left(q_{0}(y) \otimes p(x)\right) \cup r(y, z) \cup\{x=$ $w\} \vdash q_{1}(z) \otimes p(w)$. As $r \cup\{x=w\} \subseteq r[p]$ and $T$ is stable if and only if $\otimes$ is commutative, we have stationary domination and commutativity of $\widetilde{\operatorname{Inv}}(\mathfrak{U})$. For stationary equidominance and commutativity of $\overline{\operatorname{Inv}}(\mathfrak{U})$, argue analogously starting with any $r$ witnessing $q_{0}(y) \equiv_{\mathrm{D}} q_{1}(z)$.

Definition 1.26 $T$ is weakly binary iff whenever $a, b$ are tuples from some $\mathfrak{U}_{1} \succ \mathfrak{U}$ and $\operatorname{tp}(a / \mathfrak{U})$ and $\operatorname{tp}(b / \mathfrak{U})$ are invariant there is $A \subset^{+} \mathfrak{U}$ such that

$$
\operatorname{tp}(a / \mathfrak{U}) \cup \operatorname{tp}(b / \mathfrak{U}) \cup \operatorname{tp}(a, b / A) \vdash \operatorname{tp}(a, b / \mathfrak{U})
$$

Lemma 1.27 If $T$ is weakly binary and $\operatorname{tp}(a / \mathfrak{U}), \operatorname{tp}(b / \mathfrak{U})$ are both invariant, then so is $\operatorname{tp}(a b / \mathfrak{U})$.

Proof If (1) holds and $\operatorname{tp}(a / \mathfrak{U})$ and $\operatorname{tp}(b / \mathfrak{U})$ are $B$-invariant then the left-hand side of (1) is fixed by $\operatorname{Aut}(\mathfrak{U} / A B)$. As tp $(a, b / \mathfrak{U})$ is complete, it is $A B$-invariant.

Example 1.28 Every binary theory $T$, i.e. where every formula is equivalent modulo $T$ to a Boolean combination of formulas with at most two free variables, is weakly binary. This follows from the fact that $T$ is binary if and only if for any $B$ and tuples $a, b$

$$
\operatorname{tp}(a / B) \cup \operatorname{tp}(b / B) \cup \operatorname{tp}(a b / \emptyset) \vdash \operatorname{tp}(a b / B)
$$

An example of a weakly binary theory which is not binary is the theory of a dense circular order, or any other non-binary theory that becomes binary after naming some constants. A weakly binary theory which does not become binary after adding constants can be obtained by considering a structure $(M, E, R)$ where $E$ is an equivalence relation with infinitely many classes, on each class $R(x, y, z)$ is a circular order, and $R(x, y, z) \rightarrow E(x, y) \wedge E(x, z)$. The generic 3-hypergraph and $\mathrm{ACF}_{0}$ are not weakly binary. 
We thank Jan Dobrowolski for pointing out the relationship between binarity and weak binarity, therefore also implicitly suggesting a name for the latter.

Lemma 1.29 $T$ is weakly binary if and only if for every $n \geq 2$ we have the following. If $a^{0}, \ldots, a^{n-1}$ are such that for all $i<n$ we have $\operatorname{tp}\left(a^{i} / \mathfrak{U}\right) \in S^{\operatorname{inv}}(\mathfrak{U})$, then there is $A \subset^{+} \mathfrak{U}$ such that

$$
\left(\bigcup_{i=0}^{n-1} \operatorname{tp}\left(a^{i} / \mathfrak{U}\right)\right) \cup \operatorname{tp}\left(a^{0}, \ldots, a^{n-1} / A\right) \vdash \operatorname{tp}\left(a^{0}, \ldots, a^{n-1} / \mathfrak{U}\right)
$$

Proof For the nontrivial direction, assume $T$ is weakly binary. For notational simplicity we will only show the case $n=3$, and leave the easy induction to the reader. Let $a, b, c$ be tuples with invariant global type. By Lemma $1.27 \mathrm{tp}(b c / \mathfrak{U})$ is still invariant, so we can let $A$ witness weak binarity for $b, c$ and for $a, b c$ simultaneously, where $b c$ is considered now as a single tuple. Then $\operatorname{tp}(b / \mathfrak{U}) \cup \operatorname{tp}(c / \mathfrak{U}) \cup \operatorname{tp}(a, b, c / A) \vdash \operatorname{tp}(b, c / \mathfrak{U})$, and by applying weak binarity to $a, b c$ we get

$$
\begin{aligned}
& \operatorname{tp}(a / \mathfrak{U}) \cup \operatorname{tp}(b / \mathfrak{U}) \cup \operatorname{tp}(c / \mathfrak{U}) \cup \operatorname{tp}(a, b, c / A) \\
& \quad \vdash \operatorname{tp}(a / \mathfrak{U}) \cup \operatorname{tp}(b c / \mathfrak{U}) \cup \operatorname{tp}(a, b c / A) \\
& \quad \vdash \operatorname{tp}(a, b c / \mathfrak{U})
\end{aligned}
$$

Corollary 1.30 Every weakly binary theory has stationary domination and stationary equidominance.

Proof Let $p(x), q_{0}(y), q_{1}(z)$ be $A_{0}$-invariant and $r \in S_{q_{0} q_{1}}\left(A_{0}\right)$ be such that $q_{0} \cup r \vdash$ $q_{1}$. In some $\mathfrak{U}_{1}{ }^{+} \succ \mathfrak{U}$ choose $(b, c) \vDash q_{0} \cup r$, then choose $a \vDash p \mid \mathfrak{U}_{1}$. By the case $n=3$ of (2) there is some $A \subset^{+} \mathfrak{U}$, which without loss of generality includes $A_{0}$, such that

$$
\operatorname{tp}(a / \mathfrak{U}) \cup \operatorname{tp}(b / \mathfrak{U}) \cup \operatorname{tp}(c / \mathfrak{U}) \cup \operatorname{tp}(a b c / A) \vdash \operatorname{tp}(a b c / \mathfrak{U})
$$

Let $r[p]:=\operatorname{tp}_{x y z}(a b c / A) \cup\{x=w\}$ and note that $r \subseteq r[p]$. Therefore $\left(p \otimes q_{0}\right) \cup$ $r[p] \vdash q_{0} \cup r \vdash q_{1}=\operatorname{tp}(c / \mathfrak{U})$. Combining this with (3), and observing that $\operatorname{tp}(a b / \mathfrak{U})=$ $p \otimes q_{0}$, that $\operatorname{tp}(a c / \mathfrak{U})=p \otimes q_{1}$ and that $r[p] \vdash x=w$, we have

$$
\begin{aligned}
& \left(p(x) \otimes q_{0}(y)\right) \cup r[p] \\
& \quad \vdash\left(p(x) \otimes q_{0}(y)\right) \cup r[p] \cup q_{1}(z) \cup\{x=w\} \\
& \quad \vdash \operatorname{tp}_{x}(a / \mathfrak{U}) \cup \operatorname{tp}_{y}(b / \mathfrak{U}) \cup \operatorname{tp}_{z}(c / \mathfrak{U}) \cup \operatorname{tp}_{x y z}(a b c / A) \cup\{x=w\} \\
& \quad \vdash \operatorname{tp}_{w z}(a c / \mathfrak{U})=p(w) \otimes q_{1}(z)
\end{aligned}
$$

This proves stationary domination. For stationary equidominance, start with an $r$ witnessing $q_{0} \equiv_{\mathrm{D}} q_{1}$ and prove analogously that in addition $\left(p(w) \otimes q_{1}(z)\right) \cup r[p] \vdash$ $p(x) \otimes q_{0}(y)$. 
We now give some examples of ( $\left.\widetilde{\operatorname{Inv}}(\mathfrak{U}), \otimes, \geq_{D}\right)$. These characterisations can be proven with easy ad hoc arguments but, as such computations are made almost immediate by results like Proposition 3.13 or Theorem 5.11, we state them without proof. We postpone the investigation of further examples to a future paper.

Example 1.31 If $T$ is a strongly minimal theory (see Example 1.11), then $(\widetilde{\operatorname{Inv}}(\mathfrak{U}), \otimes$, $\left.\geq_{\mathrm{D}}\right) \cong(\mathbb{N},+, \geq)$.

Example 1.32 Let $T$ be the theory of an equivalence relation $E$ with infinitely many classes, all of which are infinite. Since $T$ is $\omega$-stable, by Proposition 1.25 and Proposition $1.21 \otimes$ respects $\geq_{D}$, and moreover by [13, Theorem 14.2] for every $\kappa$ there is a $\kappa$-saturated $\mathfrak{U} \vDash T$ of size $\kappa$. For such $\mathfrak{U}$ we have $\left(\widetilde{\operatorname{Inv}}(\mathfrak{U}), \otimes, \geq_{\mathrm{D}}\right) \cong \bigoplus_{\kappa} \mathbb{N}$, where each copy of $\mathbb{N}$ is equipped with the usual + and $\geq$, and $\oplus$ is the direct sum of ordered monoids.

To spell this out and give a little extra information on $\widetilde{\operatorname{Inv}}(\mathfrak{U})$ for $T$, fix a choice of representatives $\left\langle b_{i} \mid 0<i<\kappa\right\rangle$ for $\mathfrak{U} / E$ and let $\pi_{E}: \mathfrak{U} \rightarrow \mathfrak{U} / E$ be the projection to the quotient. Then an element $\llbracket p \rrbracket \in \widetilde{\operatorname{Inv}}(\mathfrak{U})$ corresponds to a $\kappa$-sequence $\left(n_{i}\right)_{i<\kappa}$ of natural numbers with finite support where, for any $c \vDash p, n_{0}=\left|\pi_{E} c \backslash \pi_{E} \mathfrak{U}\right|$ and, for positive $i, n_{i}=\left|\left\{c_{j} \in c \mid E\left(c_{j}, b_{i}\right)\right\} \backslash E\left(\mathfrak{U}, b_{i}\right)\right|$, i.e. $n_{0}$ counts the new equivalence classes represented in $p$ and, when $i$ is positive, $n_{i}$ counts the number of new points in the equivalence class of $b_{i}$. Addition is done componentwise and $\left(n_{i}\right)_{i<\kappa} \leq\left(m_{i}\right)_{i<\kappa}$ iff $\forall i<\kappa n_{i} \leq m_{i}$.

As we will see in Sect. 5, the fact that $\widetilde{\operatorname{Inv}}(\mathfrak{U})$ has the previous forms follows from the stability-theoretic properties of the theories above: Theorem 5.11 applies to both and, in the case of Example 1.31, Corollary 5.19 tells us directly that $\widetilde{\operatorname{Inv}(\mathfrak{U})} \cong \mathbb{N}$.

Example 1.33 As DLO is binary, $\otimes$ respects $\geq_{\mathrm{D}}$. We have already seen an example of two domination-equivalent types in this theory in Example 1.11. To describe $\widetilde{\operatorname{Inv}}(\mathfrak{U})$, call a cut in $\mathfrak{U}$ invariant iff it has small cofinality on exactly one side, and let IC $\mathfrak{U}$ be the set of all such. The domination-equivalence class of an invariant type in DLO is determined by the (necessarily invariant) cuts in which it concentrates and, writing $\mathscr{P}_{\text {fin }}(X)$ for the set of finite subsets of $X$, we have $\left(\widetilde{\operatorname{Inv}}(\mathfrak{U}), \otimes, \geq_{\mathrm{D}}\right) \cong\left(\mathscr{P}_{\text {fin }}(\mathrm{IC} \mathfrak{U}), \cup, \supseteq\right)$.

\section{Counterexamples}

In [3, p. 18] it was claimed without proof that $\overline{\operatorname{Inv}}(\mathfrak{U})$ is well-defined and commutative in every first-order theory. This section contains counterexamples to the statements above.

\subsection{Well-definedness}

This subsection is dedicated to the proof of the following result.

Theorem 2.1 There is a ternary, $\omega$-categorical, supersimple theory of $S U$-rank 2 with degenerate algebraic closure in which neither $\sim_{\mathrm{D}}$ nor $\equiv_{\mathrm{D}}$ are congruences with respect to $\otimes$. 
In Proposition 2.3, we present the promised theory as a Fraïssé limit (see [4, Theorem 7.1.2]) and provide an explicit axiomatisation. We then show in Proposition 2.5 that in this theory $\otimes$ does not respect $\geq_{D}$, nor $\equiv_{D}$.

Denote by $S_{3}$ the group of permutations of $\{0,1,2\}$.

Definition 2.2 Let $L$ be the relational language $L:=\left\{E^{(2)}, R_{2}^{(2)}, R_{3}^{(3)}\right\}$, where arities of symbols are indicated as superscripts, and define $\Lambda:=\Lambda_{0} \wedge \Lambda_{1}$, where

$$
\begin{aligned}
\Lambda_{0}\left(x_{0}, x_{1}, x_{2}\right) & :=\bigvee_{\sigma \in S_{3}}\left(R_{2}\left(x_{\sigma 0}, x_{\sigma 1}\right) \wedge R_{2}\left(x_{\sigma 0}, x_{\sigma 2}\right) \wedge \neg R_{2}\left(x_{\sigma 1}, x_{\sigma 2}\right)\right) \\
\Lambda_{1}\left(x_{0}, x_{1}, x_{2}\right) & :=\bigwedge_{0 \leq i<j<3} \neg E\left(x_{i}, x_{j}\right)
\end{aligned}
$$

Let $K$ be the class of finite $L$-structures where

1. $E$ is an equivalence relation,

2. $R_{2}$ is symmetric, irreflexive and $E$-equivariant, i.e. $\left(E\left(x_{0}, x_{1}\right) \wedge E\left(y_{0}, y_{1}\right)\right) \rightarrow$ $\left(R_{2}\left(x_{0}, y_{0}\right) \leftrightarrow R_{2}\left(x_{1}, y_{1}\right)\right)$,

3. $R_{3}$ is a symmetric relation, i.e. $R_{3}\left(x_{0}, x_{1}, x_{2}\right) \rightarrow \bigwedge_{\sigma \in S_{3}} R_{3}\left(x_{\sigma 0}, x_{\sigma 1}, x_{\sigma 2}\right)$, and

4. $R_{3}\left(x_{0}, x_{1}, x_{2}\right) \rightarrow \Lambda\left(x_{0}, x_{1}, x_{2}\right)$ is satisfied.

Note that in particular $R_{2}$ is still symmetric irreflexive on the quotient by $E$. We do not add an imaginary sort for this quotient; it will be notationally convenient to mention it anyway but, formally, every reference to the quotient by $E$, the relative projection, etc, is to be understood as a mere shorthand.

Proposition 2.3 1. K is a Fraïssé class with strong amalgamation.

Let $T$ be the theory of the Fraïsse limit of $K$.

2. $T$ is $\omega$-categorical, eliminates quantifiers in $L$ and has degenerate algebraic closure, i.e. for all sets $X \subseteq M \vDash T$ we have acl $X=X$.

3. $T$ is ternary, i.e. in $T$ every formula is equivalent to a Boolean combination of formulas with at most 3 free variables.

4. $T$ can be axiomatised as follows:

(I) $E$ is an equivalence relation with infinitely many classes, all of which are infinite.

(II) Whether $R_{2}\left(x_{0}, x_{1}\right)$ holds only depends on the E-classes of $x_{0}, x_{1}$; moreover, the structure induced by $R_{2}$ on the quotient by $E$ is elementarily equivalent to the Random Graph.

(III) $T$ satisfies $R_{3}\left(x_{0}, x_{1}, x_{2}\right) \rightarrow \Lambda\left(x_{0}, x_{1}, x_{2}\right)$, i.e. if $R_{3}\left(x_{0}, x_{1}, x_{2}\right)$ holds then between the $x_{i}$ there are precisely two $R_{2}$-edges and their E-classes are pairwise distinct.

(IV) Denote by $\left[x_{i}\right]_{E}$ the E-class of $x_{i}$. If $\Lambda\left(x_{0}, x_{1}, x_{2}\right)$ holds, then $R_{3}\left\lceil\left[x_{0}\right]_{E} \times\right.$ $\left[x_{1}\right]_{E} \times\left[x_{2}\right]_{E}$ is a symmetric generic tripartite 3-hypergraph, i.e. for any $i<j<3$ and $k \in\{0,1,2\} \backslash\{i, j\}$, if $U, V \subseteq\left[x_{i}\right]_{E} \times\left[x_{j}\right]_{E}$ and $U \cap V=\varnothing$ then there is $z \in\left[x_{k}\right]_{E}$ such that for every $(x, y) \in U$ we have $R_{3}(x, y, z)$ and for every $(x, y) \in V$ we have $\neg R_{3}(x, y, z)$. 
5. $T$ is supersimple of $S U$-rank 2.

Proof 1. Routine, left to the reader.

2. This is standard, see e.g. [4, Theorem 7.1.8 and Corollary 7.3.4].

3. $T$ eliminates quantifiers in a ternary relational language.

4. Easy back-and-forth between the Fraïssé limit of $K$ and any model of (I)-(IV).

5. Denote by $\pi$ the projection to the quotient by $E$. A routine application of the Kim-Pillay Theorem (see [8, Theorem 4.2]) shows that $T$ is simple and forking is given by $a \bigsqcup_{C} b \Longleftrightarrow(a \cap b \subseteq C) \wedge(\pi a \cap \pi b \subseteq \pi C)$, from which we immediately see that the SU-rank of any 1-type in $T$ is at most 2; finding a 1-type of SU-rank 2 is easy.

Definition 2.4 In $T$, define the global types

$$
\begin{aligned}
& p(x):=\left\{R_{2}(x, a) \mid a \in \mathfrak{U}\right\} \cup\left\{\neg R_{3}(x, a, b) \mid a, b \in \mathfrak{U}\right\} \\
& q_{0}(y):=\left\{\neg R_{2}(y, a) \mid a \in \mathfrak{U}\right\} \\
& q_{1}\left(z_{0}, z_{1}\right):=\left\{\neg R_{2}\left(z_{0}, a\right) \mid a \in \mathfrak{U}\right\} \cup\left\{E\left(z_{0}, z_{1}\right) \wedge z_{0} \neq z_{1}\right\}
\end{aligned}
$$

These three types are complete by quantifier elimination and the axioms of $T$ : for instance, in the case of $q_{1}$, the condition $E\left(z_{0}, z_{1}\right)$ together with the restriction of $q_{1}$ to $z_{0}$ decides all the $R_{2}$-edges of $z_{1}$, and for all $a, b \in \mathfrak{U}$ we have $\neg \Lambda_{0}\left(z_{1}, a, b\right)$, hence $\neg R_{3}\left(z_{1}, a, b\right)$. Moreover, it follows easily from their definition that $p, q_{0}$ and $q_{1}$ are all Ø-invariant.

Proposition $2.5 q_{0} \equiv_{\mathrm{D}} q_{1}$ and in particular $q_{0} \sim_{\mathrm{D}} q_{1}$. Nonetheless, $p(x) \otimes q_{0}(y) \Varangle_{\mathrm{D}}$ $p(w) \otimes q_{1}(z)$.

Proof Let $A$ be any small set and let $r\left(y, z_{0}, z_{1}\right) \in S_{q_{0} q_{1}}(A)$ contain the formula $y=z_{0}$. Clearly, $q_{1}(z) \cup r(y, z) \vdash q_{0}(y)$. Moreover, since $E\left(z_{0}, z_{1}\right) \wedge z_{0} \neq z_{1} \in\left(q_{1} \uparrow\right.$ $\emptyset) \subseteq r$ we have the first part of the conclusion.

Note that $p(x) \otimes q_{0}(y)$ is axiomatised by

$$
p(x) \cup q_{0}(y) \cup\left\{R_{2}(x, y)\right\} \cup\left\{\neg R_{3}(x, y, a) \mid a \in \mathfrak{U}\right\}
$$

and similarly $p(w) \otimes q_{1}(z)$ is axiomatised by

$$
p(w) \cup q_{1}(z) \cup\left\{R_{2}\left(w, z_{0}\right) \wedge R_{2}\left(w, z_{1}\right)\right\} \cup\left\{\neg R_{3}\left(w, z_{j}, a\right) \mid j<2, a \in \mathfrak{U}\right\}
$$

Let $A$ be any small set and $r(x, y, w, z) \in S_{p \otimes q_{0}, p \otimes q_{1}}(A)$, then pick any $a \in \mathfrak{U} \backslash A$ and $i<2$ such that $\left(p(x) \otimes q_{0}(y)\right) \cup r \vdash y \neq z_{i}$. By genericity of $R_{2}$, the set

$$
\Phi:=\left(p(x) \otimes q_{0}(y)\right) \cup r \cup\left\{R_{2}(w, a) \wedge R_{2}\left(w, z_{i}\right) \wedge \neg R_{2}\left(z_{i}, a\right)\right\}
$$

is consistent, ${ }^{3}$ and by genericity of $R_{3}$ so is $\Phi \cup\left\{R_{3}\left(w, z_{i}, a\right)\right\}$ (as well as $\Phi \cup$ $\left.\left\{\neg R_{3}\left(w, z_{i}, a\right)\right\}\right)$. This shows that

$\overline{3}$ E.g. if $r \vdash x=w \wedge y=z_{0}$ then we even have $p(x) \otimes q_{0}(y) \cup r \vdash R_{2}(w, a) \wedge R_{2}\left(w, z_{1}\right) \wedge \neg R_{2}\left(z_{1}, a\right)$. 


$$
p(x) \otimes q_{0}(y) \cup r \nvdash\left\{\neg R_{3}\left(w, z_{j}, a\right) \mid j<2, a \in \mathfrak{U}\right\} \subseteq p(w) \otimes q_{1}(z)
$$

As an aside, note that anyway $p(x) \otimes q_{0}(y) \leq_{\mathrm{D}} p(w) \otimes q_{1}(z)$ by Corollary 1.24, the map $f$ being the projection on the coordinates $\left(w, z_{0}\right)$.

Remark 2.6 An inspection of the proof shows that in fact $q_{0}$ and $q_{1}$ are "equidominancesemi-isolated" over each other (strongly $R K$-equivalent in the terminology of [16]), i.e. there is a formula $\varphi(y, z)$ consistent with $q_{0}(y) \cup q_{1}(z)$ such that $q_{0}(y) \cup\{\varphi(y, z)\} \vdash$ $q_{1}(z)$ and $q_{1}(z) \cup\{\varphi(y, z)\} \vdash q_{0}(y)$; in this case we can take $\varphi:=y=z_{0} \wedge$ $E\left(z_{0}, z_{1}\right) \wedge z_{0} \neq z_{1}$. Therefore the same counterexample also works with this finer equivalence relation.

Question 2.7 Is $\widetilde{\operatorname{Inv}}(\mathfrak{U})$ well-defined in every NIP theory?

\subsection{Commutativity}

In this subsection we prove that in the theory of the Random Graph $\widetilde{\operatorname{Inv}}(\mathfrak{U})$ coincides with $\overline{\operatorname{Inv}}(\mathfrak{U})$ and is not commutative. To begin with, note that this theory is binary, hence $\widetilde{\operatorname{Inv}}(\mathfrak{U})$ is well-defined by Corollary 1.30 and Proposition 1.21. This also follows from the characterisation of domination we are about to give in Proposition 2.11.

Definition 2.8 Let $L_{0}$ be the "empty" language, containing only equality. We say that $T$ has degenerate domination iff whenever $p(x) \geq_{\mathrm{D}} q(y)$ there is a small set $r_{0}$ of $L_{0}(\mathfrak{U})$-formulas with free variables included in $x y$ and consistent with $p$ such that $p \cup r_{0} \vdash q$.

Remark 2.9 It is easy to see that, if there is $r_{0}$ as above, then $q$ is included in $p$ up to removing realised and duplicate coordinates and renaming the remaining ones.

Lemma 2.10 Suppose $T$ has degenerate domination. Then $T$ has algebraic domination, and in particular $\otimes$ respects $\geq_{\mathrm{D}}$. Moreover for global types $p$ and $q$ the following are equivalent:

1. There is a small set $r_{0}$ of $L_{0}(\mathfrak{U})$-formulas consistent with $p \cup q$ such that $p \cup r_{0} \vdash q$ and $q \cup r_{0} \vdash p$.

2. $p \equiv \mathrm{D} q$.

3. $p \sim_{\mathrm{D}} q$.

In particular, $\otimes$ respects $\equiv_{\mathrm{D}}$ too.

Proof By Remark 2.9 degenerate domination implies algebraic domination. The implications $1 \Rightarrow 2 \Rightarrow 3$ are trivial and hold in any theory. To prove $3 \Rightarrow 1$ suppose $p(x) \sim_{\mathrm{D}} q(y)$, and let $r_{1}$ and $r_{2}$ be small sets of $L_{0}(\mathfrak{U})$-formulas with free variables included in $x y$ and consistent with $p \cup q$ such that $p \cup r_{1} \vdash q$ and $q \cup r_{2} \vdash p$. It follows easily from Remark 2.9 that we may find $r_{0}$ satisfying the same restrictions as $r_{1}$ and $r_{2}$ and such that $p \cup r_{0} \vdash q$ and $q \cup r_{0} \vdash p$ hold simultaneously.

Proposition 2.11 The Random Graph has degenerate domination. 
Proof Suppose that $r \in S_{p q}(A)$ witnesses $p(x) \geq_{\mathrm{D}} q(y)$ and assume that $q$ has no realised or duplicate coordinates. Up to a permutation of the $y_{j}$, assume that $r$ identifies $y_{0}, \ldots, y_{n-1}$ with some variables in $x$ and for all $j$ such that $n \leq j<|y|$ and all $i<|x|$ we have $r \vdash x_{i} \neq y_{j}$. If $n=|y|$ then we can let $r_{0}$ be a suitable restriction of $r$ and we are done, so assume that $n<|y|$, hence for every $i<|x|$ we have $r \vdash y_{n} \neq x_{i}$. Pick any $b \in \mathfrak{U} \backslash A$; by the Random Graph axioms $p \cup r$ is consistent with both $E\left(y_{n}, b\right)$ and $\neg E\left(y_{n}, b\right)$, contradicting $p \cup r \vdash q$.

Corollary 2.12 In the theory of the Random Graph, $\widetilde{\operatorname{Inv}}(\mathfrak{U})(=\overline{\operatorname{Inv}}(\mathfrak{U}))$ is not commutative.

Proof Consider the global types $p(x):=\{\neg E(x, a) \mid a \in \mathfrak{U}\}$ and $q(y):=\{E(y, a) \mid$ $a \in \mathfrak{U}\}$. Both are clearly Ø-invariant, and it follows straight from the definitions that $p(x) \otimes q(y) \vdash \neg E(x, y)$ and $q(y) \otimes p(x) \vdash E(x, y)$. The conclusion now follows from degenerate domination and Remark 2.9.

Other easy consequences of Proposition 2.11 are that in the theory of the Random graph

1. $\widetilde{\operatorname{Inv}}(\mathfrak{U})$ is not generated by the classes of the $n$-types for any fixed $n<\omega$,

2. $\widetilde{\operatorname{Inv}}(\mathfrak{U})$ is not generated by any family of classes of pairwise weakly orthogonal types (see Definition 3.12), and

3. for any nonrealised $p$ the submonoid generated by $\llbracket p \rrbracket$ is infinite.

Question 2.13 Let $T$ be NIP and assume $\widetilde{\operatorname{Inv}}(\mathfrak{U})$ is well-defined. Is it necessarily commutative?

The analogous question for $\overline{\operatorname{Inv}}(\mathfrak{U})$ has a negative answer. We are grateful to E. Hrushovski for pointing out the following counterexample and allowing us to include it.

Let DLOP be as in Example 1.11. It eliminates quantifiers in $\{<, P\}$, it is NIP, and it is binary, hence $\widetilde{\operatorname{Inv}}(\mathfrak{U})$ and $\overline{\operatorname{Inv}}(\mathfrak{U})$ are well-defined by Corollary 1.30 and Proposition 1.21.

Proposition 2.14 (Hrushovski) In DLOP, $\overline{\operatorname{Inv}}(\mathfrak{U})$ is not commutative.

Proof Let $p$ be the type at $+\infty$ in the predicate $P$ and $q$ the type at $+\infty$ in $\neg P$, and note that both types are $\emptyset$-invariant. Let $r \in S_{p \otimes q, q}(\emptyset)$ contain the formula $y=z$. Then $r$ witnesses $p_{x} \otimes q_{y} \equiv_{\mathrm{D}} q_{z}$, and similarly one shows that $q \otimes p \equiv_{\mathrm{D}} p$. As shown in Example 1.11, $p$ and $q$ are not equidominant, and therefore we have $(p \otimes q) \equiv_{\mathrm{D}} q \equiv_{\mathrm{D}} p \equiv_{\mathrm{D}}(q \otimes p)$.

This counterexample exploits crucially $\equiv_{\mathrm{D}}$, as opposed to $\sim_{\mathrm{D}}$. In fact, in DLOP $\widetilde{\operatorname{Inv}}(\mathfrak{U})$ is the same as in the restriction of $\mathfrak{U}$ to $\{<\}$, and in DLO $\widetilde{\operatorname{Inv}}(\mathfrak{U})$ is commutative. A further analysis also shows that $(\overline{\operatorname{Inv}}(\mathfrak{U}), \otimes)$ cannot be endowed with any order $\leq$ compatible with $\otimes$ in which $\llbracket 0 \rrbracket$ is the minimum. In fact, if $p$ and $q$ are as above, then we have already shown that $(p \otimes q) \equiv_{\mathrm{D}} q \not_{\mathrm{D}} p \equiv_{\mathrm{D}}(q \otimes p)$. If we had an order $\leq$ as above then we would get

$$
\llbracket p \rrbracket=\llbracket p \rrbracket \otimes \llbracket 0 \rrbracket \leq \llbracket p \rrbracket \otimes \llbracket q \rrbracket=\llbracket q \rrbracket=\llbracket q \rrbracket \otimes \llbracket 0 \rrbracket \leq \llbracket q \rrbracket \otimes \llbracket p \rrbracket=\llbracket p \rrbracket
$$

contradicting $\llbracket p \rrbracket \neq \llbracket q \rrbracket$. 


\section{Properties preserved by domination}

In this section we show that some properties are preserved downwards by domination. These invariants also facilitate computations of $\overline{\operatorname{Inv}}(\mathfrak{U})$ and $\overline{\operatorname{Inv}}(\mathfrak{U})$ for specific theories; an immediate consequence is for instance Corollary 3.8, that such monoids may change when passing to $T^{\mathrm{eq}}$.

The next results are related to the ones in [16], which contains a study of weak orthogonality and the global RK-order (similar to domination) in the case of generically stable regular types. Of particular interest are [16, Proposition 3.6], to which Theorem 3.5 is related, and [16, Theorem 4.4].

\subsection{Finite satisfiability, definability, generic stability}

Definition 3.1 Let $p \in S_{x}^{\mathrm{inv}}(\mathfrak{U}, A)$. A Morley sequence of $p$ over $A$ is an $A$ indiscernible sequence $\left\langle a^{i} \mid i \in I\right\rangle$, indexed on some totally ordered set $I$, such that for any $i_{0}<\cdots<i_{n-1}$ in $I$ we have $\operatorname{tp}\left(a^{i_{n-1}}, \ldots, a^{i_{0}} / A\right)=p^{(n)} \uparrow A[\mathrm{sic}]^{4}$.

Definition 3.2 Let $M \prec^{+} \mathfrak{U}$ and $A \subset^{+} \mathfrak{U}$.

1. A partial type $\pi$ is finitely satisfiable in $M$ iff for every finite conjunction $\varphi(x)$ of formulas in $\pi$ there is $m \in M$ such that $\vDash \varphi(m)$.

2. A global type $p \in S_{x}(\mathfrak{U})$ is definable over $A$ iff it is $A$-invariant and for every $\psi(x ; y) \in L$ the set $d_{p} \psi$ is clopen, i.e. of the form $\left\{q \in S_{y}(A) \mid \varphi \in q\right\}$ for a suitable $\varphi \in L(A)$.

3. A global type $p \in S_{x}(\mathfrak{U})$ is generically stable over $A$ iff it is $A$-invariant and for every ordinal $\alpha \geq \omega$ and Morley sequence $\left(a^{i} \mid i<\alpha\right)$ of $p$ over $A$, the set of formulas $\varphi(x) \in L(\mathfrak{U})$ true of all but finitely many $a^{i}$ is a complete global type.

We say that $p$ is definable iff it is definable over $A$ for some small $A$, and similarly for the other two notions.

The definition of generic stability we use is that of [1, Definition 1.6].

It is well-known (see [13, Lemma 12.10]) that every partial type which is finitely satisfiable in $M$ extends to a global type still finitely satisfiable in $M$, and that if $p \in S(\mathfrak{U})$ is finitely satisfiable in $M$ then $p$ is $M$-invariant (see [13, Theorem 12.13]). Moreover all the notions above are monotone: for instance if $p$ is generically stable over $A$ and $A \subseteq B$, then $p$ is generically stable over $B$, as Morley sequences over $B$ are in particular Morley sequences over $A$.

Fact 3.3 [14, Proposition 1(ii)] If $p$ is generically stable over a model $M$, then $p$ is finitely satisfiable in $M$.

Lemma 3.4 Suppose $p \in S_{x}^{\text {inv }}(\mathfrak{U})$ is finitely satisfiable in $M$ and $r \in S_{x y}(M)$ is consistent with $p$. Then $p \cup r$ is finitely satisfiable in $M$.

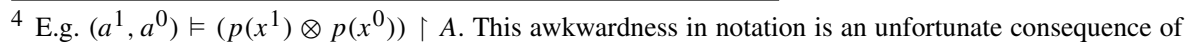
the order in which $\otimes$ is written, i.e. realising the type on the right first.
} 
Proof Pick any $\varphi(x) \in p$ and $\rho(x, y) \in r$. As $p \cup r$ is consistent, we have $p \vdash$ $\exists y(\varphi(x) \wedge \rho(x, y))$, and as $p$ is finitely satisfiable in $M$ there is $m^{0} \in M$ such that $\vDash$ $\exists y\left(\varphi\left(m^{0}\right) \wedge \rho\left(m^{0}, y\right)\right)$. In particular, $\vDash \exists y \rho\left(m^{0}, y\right)$, and since $\rho\left(m^{0}, y\right) \in L(M)$ and $M$ is a model there is $m^{1} \in M$ such that $\vDash \rho\left(m^{0}, m^{1}\right)$, so $\left(m^{0}, m^{1}\right) \vDash \varphi(x) \wedge \rho(x, y)$.

We can now prove the main result of this section. Part 3 can be seen as a generalisation of [16, Proposition 3.6]; the missing step to formally call it a generalisation would be to know that for a regular type $p$ the equivalence $p \not \perp^{\mathrm{w}} q \Leftrightarrow p \leq \mathrm{D} q$ held. To the best of the author's knowledge, this is currently only known for strongly regular generically stable types, or under additional assumptions such as stability. See [16] for the definitions of regularity and strong regularity in this context, and the next subsection for $\perp^{\mathrm{w}}$.

Theorem 3.5 Suppose $A$ is a small set such that $p_{x}, q_{y} \in S^{\text {inv }}(\mathfrak{U}, A)$ and $r \in S_{p q}(A)$ is such that $p \cup r \vdash q$.

1. If $A=M$ is a model and $p$ is finitely satisfiable in $M$, then so is $q$.

2. If $p$ is definable over $A$, then so is $q$.

3. If $A=M$ is a model and $p$ is generically stable over $M$, then so is $q$.

Proof (1) Let $\psi(y) \in q$, and let by hypothesis and compactness $\varphi(x) \in p$ and $\rho(x, y) \in r$ be such that $\vDash \forall x, y((\varphi(x) \wedge \rho(x, y)) \rightarrow \psi(y))$. Lemma 3.4 ensures the existence of $m^{0}, m^{1} \in M$ such that $\vDash \varphi\left(m^{0}\right) \wedge \rho\left(m^{0}, m^{1}\right)$, and in particular $\vDash \psi\left(m^{1}\right)$. (2) Work in $L(A)$. We want to show that for every $\psi\left(y ; z^{1}\right) \in L(A)$ the set $d_{q} \psi \subseteq$ $S_{z^{1}}(A)$ is clopen; it is sufficient to show that $d_{q} \psi$ is open, as since $\psi$ is arbitrary then the complement $d_{q}(\neg \psi)$ of $d_{q} \psi$ will be open as well. Fix $d$ such that $q \vdash \psi(y ; d)$; we are going to find a formula $\delta\left(z^{1}\right) \in \operatorname{tp}(d / A)$ such that every element of $S_{z^{1}}(A)$ satisfying $\delta$ lies in $d_{q} \psi$, proving that $\operatorname{tp}(d / A)$ is in the interior of $d_{q} \psi$.

Let $z:=z^{0} z^{1}$ and take $\varphi\left(x ; z^{0}\right) \in L(A), e \in \mathfrak{U}$ and $\rho(x, y) \in r$ such that

$$
p \vdash \underbrace{\varphi\left(x ; \underset{z^{0}}{e}\right) \wedge \forall y\left(\left(\varphi\left(x ; \underset{z^{0}}{e}\right) \wedge \rho(x, y)\right) \rightarrow \psi\left(y ; \underset{z^{1}}{d}\right)\right)}_{=: \theta(x ; e d)}
$$

As $\theta(x ; z)$ is an $L(A)$-formula and $p$ is definable over $A$, the formula $\delta\left(z^{1}\right):=$ $\left(\exists z^{0} d_{p} \theta\right)\left(z^{1}\right)$ is as well over $A$. Suppose $\tilde{d} \in \mathfrak{U}$ is such that $\vDash \delta(\tilde{d})$, and let $\tilde{e} \in \mathfrak{U}$ be such that $\vDash d_{p} \theta(\tilde{e}, \tilde{d})$. By construction we have

$$
p \vdash \varphi(x, \tilde{e}) \wedge \forall y((\varphi(x, \tilde{e}) \wedge \rho(x, y)) \rightarrow \psi(y, \tilde{d}))
$$

and it follows that $p \cup\{\rho\} \vdash \psi(y, \tilde{d})$; therefore $\psi(y, \tilde{d}) \in q$. As $\delta\left(z^{1}\right) \in \operatorname{tp}(d / A)$, we are done.

(3) Assume that $q$ is not generically stable over $M$, as witnessed by an $L(M)$-formula $\psi(y ; w)$, some $\tilde{d} \in \mathfrak{U}^{|w|}$, an ordinal $\alpha$ and a Morley sequence $\left\langle\tilde{b}^{i} \mid i<\alpha\right\rangle$ of $q$ over $M$ such that both $I:=\left\{i<\alpha \| \neg \psi\left(\tilde{b}^{i} ; \tilde{d}\right)\right\}$ and $\alpha \backslash I$ are infinite and $\psi(y ; \tilde{d}) \in q(y)$.

By Fact 3.3 and Lemma $3.4 p \cup r$ is finitely satisfiable in $M$. Since $p \cup r \vdash q$, the partial type $p \cup r \cup q$ is finitely satisfiable in $M$ as well, and therefore extends to some 
$\hat{r} \in S(\mathfrak{U})$ which is, again, finitely satisfiable in $M$, and in particular $M$-invariant; take a Morley sequence $\left\langle\left(a^{i}, b^{i}\right) \mid i \in I\right\rangle$ of $\hat{r}$ over $M$, let $f \in \operatorname{Aut}(\mathfrak{U} / M)$ be such that $f\left(\left\langle\tilde{b}^{i} \mid i \in I\right\rangle\right)=\left\langle b^{i} \mid i \in I\right\rangle$, and set $d:=f(\tilde{d})$. Note that $p, q, r$ and $\psi(y ; w)$ are fixed by $f$.

Now let $J$ be a copy of $\omega$ disjoint from $I$ and let $\left\langle a^{j} \mid j \in J\right\rangle$ realise a Morley sequence of $p$ over $M d\left\{a^{i} \mid i \in I\right\}$. We want to show that the concatenation of $\left\langle a^{i}\right|$ $i \in I\rangle$ with $\left\langle a^{j} \mid j \in J\right\rangle$ contradicts generic stability of $p$ over $M$. By construction this is a Morley sequence over $M$, and if we find $\chi(x ; d)$ such that $\vDash \chi\left(a^{i} ; d\right)$ holds for $i \in J$ but for no $i \in I$ then we are done, since $I$ and $J$ are infinite.

As $\psi(y ; d) \in q$ by $M$-invariance of $q$, there is by hypothesis $\varphi(x, y) \in r$ such that $p(x) \vdash \forall y(\varphi(x, y) \rightarrow \psi(y ; d))$. Let $\chi(x ; d)$ be the last formula. By hypothesis, for $i \in J$ we have $\vDash \chi\left(a^{i} ; d\right)$. On the other hand, for $i \in I$ we have $\left(a^{i}, b^{i}\right) \vDash$ $\varphi\left(a^{i}, b^{i}\right) \wedge \neg \psi\left(b^{i} ; d\right)$, and in particular for all $i \in I$ we have $\vDash \neg \chi\left(a^{i} ; d\right)$.

Remark 3.6 We are assuming that $p, q$ are $A$-invariant. It is not true that if $p$ is finitely satisfiable/definable/generically stable in/over some $B \subseteq A$ then $q$ must as well be such, for the same $B$. Even when $B=N \prec M=A$ are models, a counterexample can easily be obtained by taking $q$ to be the realised type of a point in $M \backslash N$.

Question 3.7 Is it true that in the setting of Remark $3.6 q$ is domination-equivalent to a type finitely satisfiable/definable/generically stable in/over $N$ ?

Corollary 3.8 There is a theory $T$ where $\widetilde{\operatorname{Inv}}(\mathfrak{U})$ changes when passing to $T^{\mathrm{eq}}$.

Proof As generic stability is preserved by domination, this happens in any theory where $T$ does not have any nonrealised generically stable type but $T^{\mathrm{eq}}$ does, as such a type cannot be domination-equivalent to any type with all variables in the home sort. An example of such a theory is that of a structure $(M,<, E)$ where $(M,<) \vDash$ DLO and $E$ is an equivalence relation with infinitely many classes, all of which are dense.

Such a thing cannot happen when passing from a stable $T$ to $T^{\mathrm{eq}}$; see Remark 5.6.

Proposition 3.9 Generically stable types commute with every invariant type.

Proof The proof of [15, Proposition 2.33] goes through even without assuming NIP provided the definition of "generically stable" is the one above.

Even if ( $\widetilde{\operatorname{Inv}}(\mathfrak{U}), \otimes)$ need not be well-defined in general, a smaller object is.

Definition 3.10 Let $\widetilde{\operatorname{Inv}}^{\mathrm{g}}(\mathfrak{U})$ be the quotient by $\sim_{\mathrm{D}}$ of the space of types which are products of generically stable types.

Corollary 3.11 ( $\left.\widetilde{\operatorname{Inv}}^{\mathrm{g}}(\mathfrak{U}), \otimes, \geq_{\mathrm{D}}\right)$ is a well-defined, commutative ordered monoid.

Proof It follows immediately from Lemma 1.14 and Proposition 3.9 that, when restricting to the set of products of generically stable types, $\sim_{\mathrm{D}}$ is a congruence with respect to $\otimes$. As the generators of $\widetilde{\operatorname{Inv}}{ }^{\mathrm{g}}(\mathfrak{U})$ commute, so does every pair of elements from it.

The reason we defined $\widetilde{I n v}^{g s}(\mathfrak{U})$ as above is that generic stability is not preserved under products: the type $p$ in [1, Example 1.7] is generically stable but $p \otimes p$ is not.

$\widetilde{I n v}^{g s}(\mathfrak{U})$ may be significantly smaller than $\widetilde{\operatorname{Inv}}(\mathfrak{U})$, and even be reduced to a single point; this happens for instance in the Random Graph, or in DLO. 


\subsection{Weak orthogonality}

Another property preserved by domination is weak orthogonality to a type. This generalises (by Proposition 5.4) a classical result in stability theory, see e.g. [10, Proposition C.13','(iii)].

Definition 3.12 We say that $p \in S_{x}(\mathfrak{U})$ and $q \in S_{y}(\mathfrak{U})$ are weakly orthogonal, and write $p \perp^{\mathrm{w}} q$, iff $p \cup q$ is a complete global type.

Note that if $p$ is invariant then $p \perp^{\mathrm{w}} q$ is equivalent to $p \cup q \vdash p \otimes q$, or in other words to the fact that for any $c \vDash q$ in some $\mathfrak{U}_{1}{ }^{+} \succ \mathfrak{U}$ we have $p \vdash p \mid \mathfrak{U} c$.

In the literature the name orthogonality is sometimes (e.g. [15, p. 136] or [16, p. 310]) used to refer to the restriction of weak orthogonality to global invariant types. We will not adopt this convention here.

Proposition 3.13 Suppose that $p_{0}, p_{1} \in S^{\mathrm{inv}}(\mathfrak{U})$ are such that $p_{0} \geq_{\mathrm{D}} p_{1}$ and $p_{0} \perp^{\mathrm{w}} q$. Then $p_{1} \perp^{\mathrm{w}} q$.

Proof Fix $\mathfrak{U}_{1}{ }^{+} \succ \mathfrak{U}$, work in its elementary diagram and suppose $p_{0}(x) \cup r(x, y) \vdash$ $p_{1}(y)$. We have to show that for any $c \in \mathfrak{U}_{1}$ realising $q$ we have $p_{1} \vdash p_{1} \mid \mathfrak{U} c$. By hypothesis, $p_{0} \vdash p_{0} \mid \mathfrak{U} c$, and by Lemma 1.13 we have $\left(p_{0} \mid \mathfrak{U} c\right) \cup r \vdash p_{1} \mid \mathfrak{U} c$, therefore $p_{0} \cup r \vdash p_{1} \mid \mathfrak{U} c$. This means that, for any $\psi(y, z) \in L(\mathfrak{U})$ such that $\psi(y, c) \in p_{1} \mid \mathfrak{U}_{c}$, there are $\varphi(x) \in p_{0}$ and $\rho(x, y) \in r$ such that $\mathfrak{U}_{1} \vDash \forall x, y((\varphi(x) \wedge$ $\rho(x, y)) \rightarrow \psi(y, c))$, therefore

$$
\mathfrak{U}_{1} \vDash \forall y((\exists x(\varphi(x) \wedge \rho(x, y))) \rightarrow \psi(y, c))
$$

As $p_{1}(y) \cup r(x, y)$ is consistent, since it is satisfied by any realisation of $p_{0}(x) \cup r(x, y)$ by hypothesis, we have $p_{1}(y) \vdash \exists x(\varphi(x) \wedge \rho(x, y))$, and the conclusion follows.

This entails the following slight generalisation of [13, Theorem 10.23].

Corollary 3.14 Let $p_{x}, q_{y} \in S^{\text {inv }}(\mathfrak{U})$. If $p \geq_{\mathrm{D}} q$ and $p \perp^{\mathrm{w}} q$, then $q$ is realised.

Proof From $p \geq_{\mathrm{D}} q$ and $p \perp^{\mathrm{w}} q$ the previous proposition gives $q \perp^{\mathrm{w}} q$. But this can only happen if $q$ is realised, otherwise $q(x) \cup q(y) \cup\{x=y\}$ and $q(x) \cup q(y) \cup\{x \neq y\}$ are both consistent.

Remark 3.15 Tanović has proved in [16, Theorem 4.4] that if $p$ is strongly regular (see [16, Definition 2.2]) and generically stable then $p$ is $\leq \mathrm{RK}$-minimal among the nonrealised types, and for all invariant $q$ we have $p \not \perp^{\mathrm{w}} q \Longleftrightarrow p \leq_{\mathrm{RK}} q$. An immediate consequence of his result and of the previous corollary is that such types are also $\leq{ }_{\mathrm{D}}$-minimal among the nonrealised types.

We conclude this section by remarking that a lot of properties are not preserved by domination-equivalence, nor by equidominance. For instance, there is an $\omega$-stable theory with two equidominant types of different Morley rank, namely $T^{\mathrm{eq}}$ where $T$ is the theory of an equivalence relation with infinitely many classes, all of which are infinite. Another property that is not preserved is having the same dp-rank, a counterexample being DLO, where if $p$ is, say, the type at $+\infty$ we have $p \equiv_{\mathrm{D}} p \otimes p$ even if the former has dp-rank 1 and the latter has dp-rank 2. 


\section{Dependence on the monster model}

In strongly minimal theories (see Example 1.31) $\widetilde{\operatorname{Inv}}(\mathfrak{U}) \cong \mathbb{N}$ regardless of $\mathfrak{U}$ while in, say, the Random Graph, $\widetilde{\operatorname{Inv}}(\mathfrak{U})$ is very close to $S^{\text {inv }}(\mathfrak{U})$ by Proposition 2.11 and the subsequent discussion: the former is obtained from the latter by identifying types that only differ because of realised, duplicate, or permuted coordinates. It is natural to ask whether and how much the quotient $\widetilde{\operatorname{Inv}}(\mathfrak{U})$ depends on $\mathfrak{U}$, and the question makes sense even when $\otimes$ does not respect $\geq_{D}$. This section investigates this matter.

\subsection{Theories with IP}

The preorder $\geq_{D}$ is the result of a series of generalisations that began in [9] with starting point the Rudin-Keisler order on ultrafilters. It is not surprising therefore that some classical arguments involving the latter object generalise as well. We show in this subsection (Proposition 4.6) that, in the case of theories with IP (see [15, Chapter 2]), one of them is the abundance of pairwise Rudin-Keisler inequivalent ultrafilters on $\mathbb{N}$; the classical proof goes through for $\sim_{D}$ as well, and shows that even the cardinality of $\widetilde{\operatorname{Inv}}(\mathfrak{U})$ depends on $\mathfrak{U}$.

In this subsection $\llbracket p \rrbracket$ stands for the $\sim_{\mathrm{D}}$-class of $p$. Even if we state everything for $\sim_{\mathrm{D}}$ and its quotient $\operatorname{Inv}(\mathfrak{U})$, the same arguments work if we replace $\sim_{\mathrm{D}}$ by $\equiv_{\mathrm{D}}, \widetilde{\operatorname{Inv}}(\mathfrak{U})$ by $\overline{\operatorname{Inv}}(\mathfrak{U})$ and interpret $\llbracket p \rrbracket$ as the class of $p$ modulo $\equiv_{\mathrm{D}}$.

The following result is classical, see e.g. [4, Exercise 4(a) of Section 10.1 and Theorem 10.2.1].

Fact 4.1 Let $T$ be any theory and $\lambda \geq|T|$. Then $T$ has a $\lambda^{+}$-saturated and $\lambda^{+}$-strongly homogeneous model of cardinality at most $2^{\lambda}$.

For the rest of this subsection, let $\mathfrak{U}$ be $\lambda^{+}$-saturated and $\lambda^{+}$-strongly homogeneous of cardinality at most $2^{\lambda}$, let $\sigma$ be the least cardinal such that $\mathfrak{U}$ is not $\sigma^{+}$-saturated, and let $\kappa=|\mathfrak{U}|$. Thus $\lambda^{+} \leq \sigma \leq \kappa \leq 2^{\lambda}$.

Lemma 4.2 In the notations above, for every $p \in S^{\text {inv }}(\mathfrak{U})$ we have $|\llbracket p \rrbracket| \leq \mid\left\{q \mid q \leq{ }_{\mathrm{D}}\right.$ $p\} \mid \leq \kappa^{<\sigma}$.

Proof Clearly $\llbracket p \rrbracket \subseteq\left\{q \mid q \leq_{\mathrm{D}} p\right\}$. For every $q \leq_{\mathrm{D}} p$, there is some small $r_{q}$ such that $p \cup r_{q} \vdash q$. If $r_{q}=r_{q^{\prime}}$ then $q=q^{\prime}$, and therefore $|\{q \mid q \leq \mathrm{D} p\}|$ is bounded by the number of small types. As "small" means of cardinality strictly less than $\sigma$, the number of such types is at most the size of $\bigcup_{A \subset \mathfrak{U},|A|<\sigma} S(A)$, which cannot exceed $\kappa^{<\sigma} \cdot 2^{<\sigma}=\kappa^{<\sigma}$.

Corollary 4.3 The same bound applies to sets of the form $\left\{\llbracket q \rrbracket \mid \llbracket q \rrbracket \leq_{\mathrm{D}} \llbracket p \rrbracket\right\}$, for $a$ fixed $p$.

Lemma 4.4 If $T$ has IP, then $2^{\lambda}=\kappa=2^{<\sigma}=\kappa^{<\sigma}$.

Proof If $\varphi(x ; y)$ witnesses IP, then over a suitable model of cardinality $\lambda$, which we may assume to be embedded in $\mathfrak{U}$, there are $2^{\lambda}$-many $\varphi$-types, and a fortiori types. This gives the first equality, and the same argument with any $\mu$ such that $\lambda \leq \mu<\sigma$ gives the second one. The third one follows by cardinal arithmetic. 
Recall the following property of theories with IP.

Fact 4.5 If $T$ has IP, then for every $\lambda \geq|T|$ there is a type $p$ over some $M \vDash T$ such that $|M|=\lambda$ and $p$ has $2^{2^{\lambda}}$-many $M$-invariant extensions. Moreover, such extensions can be chosen to be over any $\lambda^{+}$-saturated model.

Proof This is [13, Theorem 12.28]. The "moreover" part follows from the proof in the referenced source: in its notation, it is enough to realise the $f$-types of the $b_{w}$ over $\left\{a_{\alpha} \mid \alpha<\lambda\right\}$.

Proposition 4.6 If $T$ has IP and $\mathfrak{U}$ is $\lambda^{+}$-saturated and $\lambda^{+}$-strongly homogeneous of cardinality $2^{\lambda}$, then $\widetilde{\operatorname{Inv}}(\mathfrak{U})$ has size $2^{|\mathfrak{U}|}$.

Proof Since $\lambda^{+}$-saturation implies $\lambda^{+}$-universality, we may assume that the $M$ given by Fact 4.5 is an elementary submodel of $\mathfrak{U}$, and by the "moreover" part of Fact 4.5 we have $\left|S^{\text {inv }}(\mathfrak{U})\right| \geq 2^{2^{\lambda}}$. But then by Lemma 4.2

$$
2^{\kappa}=2^{2^{\lambda}} \leq\left|S^{\mathrm{inv}}(\mathfrak{U})\right|=\sum_{\llbracket p \rrbracket \in \widetilde{\operatorname{nnv}}(\mathfrak{U})}|\llbracket p \rrbracket| \leq|\widetilde{\operatorname{Inv}}(\mathfrak{U})| \cdot \kappa^{<\sigma}
$$

Using Lemma 4.4 we obtain $2^{\kappa} \leq|\widetilde{\operatorname{Inv}}(\mathfrak{U})| \cdot \kappa$, and therefore $|\widetilde{\operatorname{Inv}}(\mathfrak{U})|=2^{\kappa}$.

Corollary 4.7 If T has IP then $\widetilde{\operatorname{Inv}}(\mathfrak{U})$ depends on $\mathfrak{U}$.

Proof If $\mathfrak{U}_{1}$ is, say, $\left|\mathfrak{U}_{0}\right|^{+}$-saturated of cardinality $2^{\left|\mathfrak{U}_{0}\right|}$, then $\left|\widetilde{\operatorname{Inv}}\left(\mathfrak{U}_{1}\right)\right|=2^{2^{\left|\mathfrak{U}_{0}\right|}}$.

Question 4.8 Is there an unstable NIP theory where $\widetilde{\operatorname{Inv}}(\mathfrak{U})$ does not depend on $\mathfrak{U}$ ? Is there one where $\overline{\operatorname{Inv}}(\mathfrak{U})$ does not depend on $\mathfrak{U}$ ?

Question 4.9 Can $\widetilde{\operatorname{Inv}}(\mathfrak{U})$ or $\overline{\operatorname{Inv}}(\mathfrak{U})$ be finite?

By the results above and Proposition $5.20^{5}$ it is enough to consider the NIP unstable case.

\subsection{The map $\mathfrak{e}$}

Let $\mathfrak{U}_{1}{ }^{+} \succ \mathfrak{U}_{0}$. The map $p \mapsto p \mid \mathfrak{U}_{1}$ shows that, for every tuple of variables $x$, a copy of $S_{x}^{\text {inv }}\left(\mathfrak{U}_{0}\right)$ sits inside $S_{x}^{\text {inv }}\left(\mathfrak{U}_{1}\right)$; for instance, if $T$ is stable, this is nothing more than the classic identification of types over $\mathfrak{U}_{0}$ with types over $\mathfrak{U}_{1}$ that do not fork over $\mathfrak{U}_{0}$.

Definition 4.10 If $\mathfrak{U}_{0} \prec^{+} \mathfrak{U}_{1}$, we define the map $\mathfrak{e}: \widetilde{\operatorname{Inv}}\left(\mathfrak{U}_{0}\right) \rightarrow \widetilde{\operatorname{Inv}}\left(\mathfrak{U}_{1}\right)$ as $\mathfrak{e}(\llbracket p \rrbracket):=$ $\llbracket p \mid \mathfrak{U}_{1} \rrbracket$.

Proposition 4.11 The map $\mathfrak{e}$ is well-defined and weakly increasing. If moreover $\otimes$ respects $\geq_{\mathrm{D}}$, then $\mathfrak{e}$ is also a homomorphism of monoids.

$\overline{5}$.... and the fact that we only consider theories with no finite models... 
Proof If $p \geq_{\mathrm{D}} q$, as witnessed by $r$, by Lemma 1.13 we have $\left(p \mid \mathfrak{U}_{1}\right) \cup r \vdash\left(q \mid \mathfrak{U}_{1}\right)$, and the first part follows.

Suppose now that $\otimes$ respects $\geq_{\mathrm{D}}$ and denote for brevity $p \mid \mathfrak{U}_{1}$ with $\tilde{p}$. Recall that, if $\varphi(x, y) \in L\left(\mathfrak{U}_{0}\right)$, then $\varphi \in p_{x} \otimes q_{y}$ if and only if for any $b \vDash q$ we have $\varphi(x, b) \in$ $p \mid \mathfrak{U}_{0} b$. This in particular holds for any $b \vDash \widetilde{q}$ and shows that $(\tilde{p} \otimes \widetilde{q}) \uparrow \mathfrak{U}_{0}=p \otimes q$, or in other words $(p \otimes q) \mid \mathfrak{U}_{1}=\tilde{p} \otimes \tilde{q}$. Therefore

$$
\mathfrak{e}(\llbracket p \rrbracket) \otimes \mathfrak{e}(\llbracket q \rrbracket)=\llbracket \widetilde{p} \rrbracket \otimes \llbracket \widetilde{q} \rrbracket=\llbracket(p \otimes q) \mid \mathfrak{U}_{1} \rrbracket=\mathfrak{e}(\llbracket p \otimes q \rrbracket)
$$

so $\mathfrak{e}$ is a homomorphism of semigroups. As $\mathfrak{e}$ clearly sends $\llbracket 0 \rrbracket$ to $\llbracket 0 \rrbracket$, because an extension of a realised type is realised, we have the conclusion.

Lemma 4.12 Suppose that every time $p, q \in S\left(\mathfrak{U}_{1}\right)$ are $A_{0}$-invariant for some $A_{0} \subset^{+}$ $\mathfrak{U}_{0}$ and $p \geq_{\mathrm{D}} q$ then this can be witnessed by some $r^{\prime} \in S\left(A^{\prime}\right)$ such that $\mathfrak{U}_{0}$ is $\left|A^{\prime}\right|^{+}$. saturated and $\left|A^{\prime}\right|^{+}$-strongly homogeneous. ${ }^{6}$ Then $\mathfrak{e}$ is injective and $\mathfrak{e}(\llbracket p \rrbracket) \geq_{\mathrm{D}} \mathfrak{e}(\llbracket q \rrbracket)$ implies $\llbracket p \rrbracket \geq \mathrm{D} \llbracket q \rrbracket$.

Proof We have to check that, in the previous notations, if $\tilde{p} \geq_{\mathrm{D}} \widetilde{q}$ then $p \geq_{\mathrm{D}} q$. If $\widetilde{p} \geq_{\mathrm{D}} \widetilde{q}$ can be witnessed by some $r$ with parameters in some $A \subset^{+} \mathfrak{U}_{0}$, then we are done: by Lemma $1.12 p \cup r \vdash q$.

As $\mathfrak{U}_{0}$ is $\left|A_{0} \cup A^{\prime}\right|^{+}$-saturated and $\left|A_{0} \cup A^{\prime}\right|^{+}$-strongly homogeneous, up to taking unions we may assume $A^{\prime} \supseteq A_{0}$, and by hypothesis we can find an $A_{0}$-isomorphic copy $A$ of $A^{\prime}$ inside $\mathfrak{U}_{0}$. Let $f \in \operatorname{Aut}\left(\mathfrak{U}_{1} / A_{0}\right)$ be such that $A=f\left(A^{\prime}\right)$ and define

$$
\mathfrak{U}_{0}^{\prime}:=f^{-1}\left(\mathfrak{U}_{0}\right) \quad p^{\prime}:=f^{-1}(p) \in S\left(\mathfrak{U}_{0}^{\prime}\right) \quad q^{\prime}:=f^{-1}(q) \in S\left(\mathfrak{U}_{0}^{\prime}\right)
$$

As $\widetilde{p}$ and $\widetilde{q}$ are $A_{0}$-invariant they are fixed by $f$, so $p^{\prime} \subseteq \widetilde{p}$ and $q^{\prime} \subseteq \widetilde{q}$; by Lemma 1.12 we therefore have $p^{\prime} \cup r^{\prime} \vdash q^{\prime}$, and so $r:=f\left(r^{\prime}\right)$ witnesses both $\tilde{p} \geq_{\mathrm{D}} \tilde{q}$ and $p \geq \mathrm{D} q$.

The hypotheses of the lemma are satisfied for instance if $T$ has degenerate domination, or if $T$ is stable by Corollary 5.5. Note that, should $\mathfrak{e}$ fail to be injective, we could still in principle have two monster models $\mathfrak{U}_{0}$ and $\mathfrak{U}_{1}$ of different cardinalities such that $\left|\widetilde{\operatorname{Inv}}\left(\mathfrak{U}_{0}\right)\right|=\left|\widetilde{\operatorname{Inv}}\left(\mathfrak{U}_{1}\right)\right|$. For instance, even in a theory with IP, the results of the previous subsection do not prevent this from happening in the case where $\left|\mathfrak{U}_{0}\right|$ and $\left|\mathfrak{U}_{1}\right|$ are, say, strongly inaccessible cardinals.

Question 4.13 Is the image of $\mathfrak{e}$ downward closed? More generally, if $p \in S^{\text {inv }}(\mathfrak{U})$ is dominated by some $M$-invariant type, is $p$ then domination-equivalent to some $M$-invariant type?

By standard results (see [15, Lemma 2.18] and [1, Fact 1.9(2)]), if Question 4.13 has a positive answer then so does Question 3.7; for instance, any $M$-invariant type finitely satisfiable in some small $N$ is finitely satisfiable in $M$.

\footnotetext{
6 Note that $A^{\prime}$ need not be a subset of $\mathfrak{U}_{0}$.
} 


\section{Stable theories}

The domination preorder we defined generalises a notion from classical stability theory. For the sake of completeness, we collect in this section what is already known in the stable case. From now on, we will assume some knowledge of stability theory from the reader, and $T$ will be stable unless otherwise stated; we repeat this assumption for emphasis. References for almost everything that follows can be found in e.g. $[2,12,13] .{ }^{7}$ In this section, we mention orthogonality of types, denoted by $\perp$, which is a strengthening of weak orthogonality that can be defined in a stable theory for stationary types (see [12, Section 1.4.3]). For global types, it coincides with weak orthogonality.

\subsection{The classical definition}

In the following definition $A$ is allowed to be a large set, e.g. we allow $A=\mathfrak{U}$.

Definition 5.1 We say that a weakly dominates $b$ over $A$ iff for all $d$ we have $a \downarrow_{A}$ $d \Longrightarrow b \perp_{A} d$. We say that $a$ dominates $b$ over $A$, written $a \triangleright_{A} b$, iff for every $B \supseteq A$ if $a b \bigsqcup_{A} B$ then $a$ weakly dominates $b$ over $B$.

Fact 5.2 (See [12, Lemma 1.4.3.4] and [13, Lemma 19.18]) Suppose $A \subseteq B$ and $a b \downarrow_{A} B$. Then $a \triangleright_{B} b$ if and only if $a \triangleright_{A} b$. Moreover, over a $|T|^{+}$-saturated model domination and weak domination are equivalent.

Definition 5.3 For stationary $p, q \in S(A)$ we say that $p \triangleright q$ iff there are $a \vDash p$ and $b \vDash q$ such that $a \triangleright_{A} b$. If $p \triangleright q \triangleright p$ we write $p \bowtie q$. If there are $a \vDash p$ and $b \vDash q$ such that $a \triangleright_{A} b \triangleright_{A} a$ we write $p \doteq q$.

Proposition 5.4 Suppose that $T$ is stable and $p, q$ are global types. Then

1. $p \geq_{\mathrm{D}} q$ if and only if $p \triangleright q$.

2. $p \equiv_{\mathrm{D}} q$ if and only if $p \doteq q$.

3. If $p \geq_{\mathrm{D}} q$ then this is witnessed by some $r \in S_{p q}(M)$ with $|M| \leq|T|$.

Proof sketch If $p \cup r \vdash q$, where $r \in S(M)$, and $(a, b) \vDash r$, then it can be checked that $a$ weakly dominates $b$ over $M$, and if $M$ is large enough this yields $p \triangleright q$. In the other direction, take $a \vDash p, b \vDash q$ witnessing domination and consider their type over some $M$ of size at most $|T|$ such that $a b \downarrow_{M} \mathfrak{U}$. The rest follows easily. For more details, see e.g. [12, Lemma 1.4.3.4 (iii)].

More conceptual proofs of the first and last point can be obtained from the classical results that $p \triangleright q$ if and only if $q$ is realised in the prime a-model containing a realisation of $p$, and that prime a-models are a-atomic (see [12, Lemma 1.4.2.4]). Note that a consequence of this equivalence is that in a stable theory semi-a-isolation (i.e. $\geq_{\mathrm{D}}$ by point 3 of the previous Proposition) is the same as a-isolation: if $p \cup r \vdash q$ then $r$ can be chosen such that $p \cup r$ is complete, despite $r$ being small.

\footnotetext{
7 In [2], some results are only stated for theories with regular $\kappa(T)$; the reason for this is that [2] defines an a-model to be a strongly $\kappa(T)$-saturated model, as opposed to a strongly $\kappa_{\mathrm{r}}(T)$-saturated one.
} 
Corollary 5.5 If $T$ is stable, then $\mathfrak{e}$ is injective and $\mathfrak{e}(\llbracket p \rrbracket) \geq_{\mathrm{D}} \mathfrak{e}(\llbracket q \rrbracket)$ implies $\llbracket p \rrbracket \geq_{\mathrm{D}}$ $\llbracket q \rrbracket$.

Proof By point 3 of Proposition 5.4 we can apply Lemma 4.12.

Remark 5.6 While studying $\widetilde{\operatorname{Inv}}(\mathfrak{U})$ in a stable $T$, there is no harm in passing to $T^{\mathrm{eq}}$, which we see as a multi-sorted structure, for the following reason. Even without assuming stability, every type $p \in S(\mathfrak{U})$ in $T^{\text {eq }}$ is dominated by, and in particular (if it is nonrealised) not weakly orthogonal to, a type $q \in S(\mathfrak{U})$ with all variables in the home sort via the projection map. Suppose now that $T$ is stable and let $M$ be such that $p$ and $q$ do not fork over $M$. By (the proof of) [13, Lemma 19.21] there is a (possibly forking) extension of $q \uparrow M$ which is equidominant with $p$. Trivially, this extension has all variables in the home sort. We would like to thank Anand Pillay for pointing this out.

Remark 5.7 Definition 5.1 makes sense also in simple theories, and more generally in rosy theories if we replace forking by p-forking (see [11]). One can then give a definition of $\triangleright$ even for types that are not stationary but, in the unstable case, even for global types the relation $\triangleright$ need not coincide with $\geq_{D}$. For instance, in the notation of Definition 2.4, let $(b, c) \vDash q_{1}$ and $a \vDash p \mid \mathfrak{U} b c$, and recall that in $T$ forking is characterised as

$$
e \underset{C}{\perp} d \Longleftrightarrow(e \cap d \subseteq C) \wedge(\pi e \cap \pi d \subseteq \pi C)
$$

It follows that, for all $B \supseteq \mathfrak{U}$ such that $a b c \bigsqcup_{\mathfrak{U}} B$, and for all $d$ such that $a b \bigsqcup_{B} d$, we have $a b c \perp_{B} d$, and therefore $a b \triangleright_{\mathfrak{U}} a b c$. Since $\operatorname{tp}(a, b / \mathfrak{U})=p \otimes q_{0}$ and $\operatorname{tp}(a, b c / \mathfrak{U})=p \otimes q_{1}$ this shows $p \otimes q_{0} \triangleright p \otimes q_{1}$, but by Proposition $2.5 p \otimes q_{0} \geq_{\mathrm{D}}$ $p \otimes q_{1}$.

\subsection{Thin theories}

Recall that a stable theory is thin iff every complete type has finite weight (see [12, Section 1.4.4] for the definition of weight). For instance superstable theories are thin ([12, Corollary 1.4.5.8]) and so are theories with no dense forking chains ([12, Lemma 4.3.7]) or where every complete type has rudimentarily finite weight ([12, Proposition 4.3.10]). This hypothesis provides a structure theorem for $\widetilde{\operatorname{Inv}}(\mathfrak{U})$, namely Theorem 5.11. This result is implicit in the literature (see [12, Proposition 4.3.10]), but we need to state it is as done below for later use.

Fact 5.8 [12, Lemma 1.4.4.2] If $p$ and $q$ have both weight 1 then the following are equivalent: 1. $p \not \perp q$. 2. $p \sim_{\mathrm{D}} q$. 3. $p \equiv_{\mathrm{D}} q$.

Fact 5.9 [2, Lemma 5.6.4 (iv)] Weight is preserved by domination-equivalence.

Lemma 5.10 If $p$ has weight $w(p)=1$, then the monoid generated by $\llbracket p \rrbracket$ in $\widetilde{\operatorname{Inv}}(\mathfrak{U})$ is isomorphic to $\mathbb{N}$. 
Proof Since weight is additive over $\otimes\left([2\right.$, Proposition 5.6.5 (ii)] $)$ we have $w\left(p^{(n)}\right)=n$ and we conclude by Fact 5.9 that the map $n \mapsto \llbracket p^{(n)} \rrbracket$ is an isomorphism between $\mathbb{N}$ and the monoid generated by $\llbracket p \rrbracket$.

Theorem 5.11 If $T$ is thin, then there are a cardinal $\kappa$, possibly depending on $\mathfrak{U}$, and an isomorphism $f: \widetilde{\operatorname{Inv}}(\mathfrak{U}) \rightarrow \bigoplus_{\kappa} \mathbb{N}$. Moreover, $p \perp q$ if and only if $f(p)$ and $f(q)$ have disjoint supports.

Proof Let $\left\langle\llbracket p_{i} \rrbracket \mid i<\kappa\right\rangle$ be an enumeration without repetitions of the $\sim_{\mathrm{D}^{-}}$classes of types of weight 1 . For such classes, define $f\left(\llbracket p_{i} \rrbracket\right)$ to be the characteristic function of $\{i\}$, then extend $f$ to classes of products of weight-one types by sending $\llbracket p \otimes q \rrbracket$ to $f(\llbracket p \rrbracket)+f(\llbracket q \rrbracket)$ and $\llbracket 0 \rrbracket$ to the function which is constantly 0 . It is easy to show using Fact 5.8 and Corollary 3.14 that $f$ is well-defined, i.e. does not depend on the decomposition as product of weight-one types, and that $f$ is injective. By [12, Proposition 4.3.10] in a thin theory every type is domination-equivalent to a finite product of weight-one types, so $f$ is defined on the whole of $\widetilde{\operatorname{Inv}}(\mathfrak{U})$. By Lemma 5.10 if $w(p)=1$ then the monoid generated by $\llbracket p \rrbracket$ is isomorphic to $\mathbb{N}$ and this easily entails that $f$ is surjective. It is also clear that $f$ is an isomorphism of ordered monoids. Since two types of weight 1 are either weakly orthogonal or domination-equivalent by Fact 5.8 and, by [10, Proposition C.5(i)], in stable theories $p \perp q_{0} \otimes q_{1}$ if and only if $p \perp q_{0}$ and $p \perp q_{1}$, the last statement follows.

Remark 5.12 Weight, which is preserved by domination-equivalence (Fact 5.9), can, in the thin case, be read off $f(\widetilde{\operatorname{Inv}}(\mathfrak{U}))$ by taking "norms". Specifically, if $f(\llbracket p \rrbracket)=$ $\left(n_{i}\right)_{i<\kappa}$, then $w(p)=\sum_{i<\kappa} n_{i}$ (recall that every $\left(n_{i}\right)_{i<\kappa} \in \bigoplus_{\kappa} \mathbb{N}$ has finite support).

Proposition 5.13 If $T$ is thin, then $\equiv_{\mathrm{D}}$ and $\sim_{\mathrm{D}}$ coincide.

Proof By [7, Theorem 4.4.10] every type is in fact equidominant with a finite product of types of weight 1 . The conclusion then follows from Fact 5.8 and the fact that, as $T$ is stable, $\otimes$ respects both $\sim_{\mathrm{D}}$ and $\equiv_{\mathrm{D}}$.

\subsection{Dimensionality and dependence on the monster}

At least in the thin case some classical results imply that independence of $\widetilde{\operatorname{Inv}}(\mathfrak{U})$ from the choice of $\mathfrak{U}$ is equivalent to dimensionality of $T$, also called nonmultidimensionality.

Definition 5.14 Let $T$ be stable. We say that $T$ is dimensional iff for every nonrealised global type $p$ there is a global type $q$ that does not fork over $\emptyset$ and such that $p \not \perp q$. We say that $T$ is bounded iff $|\widetilde{\operatorname{Inv}}(\mathfrak{U})|<|\mathfrak{U}|$.

If $T$ is thin, then $T$ is dimensional if and only if it is bounded, as follows e.g. from Theorem 5.11 (alternatively, see the proof of [2, Lemma 7.1.2], but replace "superstable" with "thin" and "regular types" with "weight-one types"). In this case the number of copies of $\mathbb{N}$ required is bounded by $2^{|T|}$, and by $|T|$ if $T$ is totally transcendental, see e.g. [2, Corollary 7.1.1]. In fact, some sources define boundedness only for superstable theories, essentially as boundedness of the number of copies of $\mathbb{N}$ given by Theorem 5.11 . 
Conjecture 5.15 Let $T$ be stable. The following are equivalent: 1. T is bounded. 2. T is dimensional. 3. e is surjective.

$1 \Rightarrow 2$ follows from [2, Proposition 5.6.2] and $3 \Rightarrow 1$ is trivial, so it remains to prove $2 \Rightarrow 3$, namely that if there is a type over $\mathfrak{U}_{1}$ not domination-equivalent to any type that does not fork over $\mathfrak{U}_{0}$, then there is a type orthogonal to every type that does not fork over $\emptyset$.

Proposition 5.16 If $T$ is thin then Conjecture 5.15 holds.

Proof Suppose $\mathfrak{U}_{0} \prec^{+} \mathfrak{U}_{1}$ and let $f_{j}: \widetilde{\operatorname{Inv}}\left(\mathfrak{U}_{j}\right) \rightarrow \bigoplus_{\kappa_{j}} \mathbb{N}$, for $j \in\{0,1\}$, be given by Theorem 5.11. Let

$$
g:=f_{1} \circ \mathfrak{e} \circ f_{0}^{-1}: \bigoplus_{\kappa_{0}} \mathbb{N} \rightarrow \bigoplus_{\kappa_{1}} \mathbb{N}
$$

Since weight is preserved by nonforking extensions (e.g. by [2, Definition 5.6.6 (iii)]), $\mathfrak{e}$ sends types of weight 1 to types of weight 1 . Therefore by Remark 5.12 we may decompose the codomain of $g$ as

$$
\bigoplus_{\kappa_{1}} \mathbb{N} \cong \bigoplus_{i<\kappa_{0}} \mathbb{N} \oplus \underset{\kappa_{0} \leq i<\kappa_{1}}{\bigoplus} \mathbb{N}
$$

where the direct summand $\bigoplus_{i<\kappa_{0}} \mathbb{N}$ may be assumed to coincide with Im $g$. It then follows that if $\mathfrak{e}$ is not surjective then we can find $\llbracket p \rrbracket \notin \operatorname{Im} \mathfrak{e}$ such that $p$ has weight 1 . Again by Theorem 5.11, such a $p$ needs to be orthogonal to every type in the union of $\operatorname{Im} \mathfrak{e}$, which is the set of types that do not fork over $\mathfrak{U}_{0}$. In particular, $p$ is orthogonal to every type that does not fork over $\emptyset$.

A possible attack in the general case could be, assuming $\mathfrak{e}$ is not surjective, to try to find a type of weight 1 outside of its image. This will be either orthogonal to every type that does not fork over $\mathfrak{U}$, or dominated by one of them by [2, Corollary 5.6.5]. If we knew a positive answer to Question 4.13 at least in the stable case, and if we managed to find a type as above, then we would be done.

A possibly related notion is the strong compulsion property (see [6, Definition 2]); it implies that every type over $\mathfrak{U}_{1}{ }^{+} \succ \mathfrak{U}_{0}$ is either orthogonal to $\mathfrak{U}_{0}$ or dominates a type that does not fork over it. Whether all countable stable $T^{\mathrm{eq}}$ have a weakening of this property is [6, Conjecture 18].

We conclude with two easy consequences of some classical results.

Definition 5.17 A stable theory $T$ is unidimensional iff whenever $p \perp q$ at least one between $p$ and $q$ is algebraic.

If $T$ is totally transcendental then unidimensionality is the same as categoricity in every cardinality strictly greater than $|T|$ (see [2, Proposition 7.1.1]). Unidimensional theories may still fail to be totally transcendental, e.g. $\mathrm{Th}(\mathbb{Z},+)$ is such. Anyway, the following classical theorem by Hrushovski (see [5, Theorem 4]) tells us that the situation cannot be much worse than that. 
Theorem 5.18 (Hrushovski) Every unidimensional theory is superstable.

Corollary 5.19 A stable $T$ is unidimensional if and only if $\widetilde{\operatorname{Inv}}(\mathfrak{U}) \cong \mathbb{N}$.

Proof If $T$ is unidimensional, by Hrushovski's result we have the hypothesis of Theorem 5.11, and the conclusion then follows easily from the definition of unidimensionality. In the other direction, the hypothesis yields that any two types are

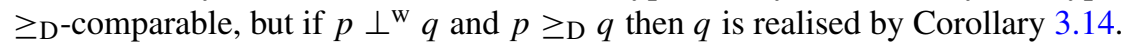

Compare the previous corollary with [9, Proposition 5]. Note that the hypothesis that $T$ is stable is necessary: in the random graph if $p \perp^{\mathrm{w}} q$ then one between $p$ and $q$ must be algebraic, but $\widetilde{\operatorname{Inv}}(\mathfrak{U})$ is not commutative by Corollary 2.12 .

Proposition 5.20 If $T$ is stable then $\mathbb{N}$ embeds in $\widetilde{\operatorname{Inv}}(\mathfrak{U})$.

Proof By [13, Lemma 13.3 and p. 336] in any stable theory there is always a type $p$ of U-rank 1, and in particular of weight $w(p)=1$ (see [13, before Theorem 19.9]). The conclusion follows from Lemma 5.10.

Acknowledgements First of all, I would like to thank my supervisors, Dugald Macpherson and Vincenzo Mantova, whose guidance, suggestions and feedback have been more than invaluable. I am also indebted to Jan Dobrowolski for the useful discussions on binary theories, Ehud Hrushovski for his permission to include Proposition 2.14, and Anand Pillay for pointing out Remark 5.6. Part of this research was carried out while I was participating in the thematic trimester Model Theory, Combinatorics and Valued Fields held in Paris from January to April 2018. I would like to thank the organising committee for making the trimester possible, and the Institut Henri Poincaré, the Centre National de la Recherche Scientifique and the School of Mathematics of the University of Leeds for supporting financially my participation in it. This research is part of the author's Ph.D. project, supported by a Leeds Anniversary Research Scholarship.

Open Access This article is distributed under the terms of the Creative Commons Attribution 4.0 International License (http://creativecommons.org/licenses/by/4.0/), which permits unrestricted use, distribution, and reproduction in any medium, provided you give appropriate credit to the original author(s) and the source, provide a link to the Creative Commons license, and indicate if changes were made.

\section{References}

1. Adler, H., Casanovas, E., Pillay, A.: Generic stability and stability (2012). arXiv:1210.5957v1

2. Buechler, S.: Essential Stability Theory, volume 4 of Perspectives in Logic. Cambridge University Press, Cambridge (2017)

3. Haskell, D., Hrushovski, E., Macpherson, D.: Stable Domination and Independence in Algebraically Closed Valued Fields, volume 30 of Lecture Notes in Logic. Cambridge University Press, Cambridge (2008)

4. Hodges, W.: Model Theory, volume 42 of Encyclopedia of Mathematics and its Applications. Cambridge University Press, Cambridge (1993)

5. Hrushovski, E.: Unidimensional theories are superstable. Ann. Pure Appl. Log. 50, 117-137 (1990)

6. Hyttinen, T.: Remarks on structure theorems for $\omega_{1}$-saturated models. Notre Dame J. Form. Log. 36, 269-278 (1995)

7. Kim, B.: Simplicity Theory, volume 53 of Oxford Logic Guides. Oxford University Press, Oxford (2014)

8. Kim, B., Pillay, A.: Simple theories. Ann. Pure Appl. Log. 88, 149-164 (1997)

9. Lascar, D.: Généralisation de l'ordre de Rudin-Keisler aux types d'une théorie. In Colloques internationaux du CNRS, vol 249, pp. 73-81. Éd. du CNRS (1975)

10. Makkai, M.: A survey of basic stability theory, with particular emphasis on orthogonality and regular types. Isr. J. Math. 49, 181-238 (1984) 
11. Onshuus, A., Usvyatsov, A.: Thorn orthogonality and domination in unstable theories. Fundam. Math. 214, 241-268 (2011)

12. Pillay, A.: Geometric Stability Theory, volume 32 of Oxford Logic Guides. Oxford University Press, Oxford (1996)

13. Poizat, B.: A Course in Model Theory. Universitext. Springer, Berlin (2000)

14. Pillay, A., Tanović, P.: Generic stability, regularity, and quasiminimality. In Models, Logics and Higherdimensional Categories, volume 53 of CRM Proceedings and Lecture Notes, pp. 189-212. American Mathematical Society (2009)

15. Simon, P.: A Guide to NIP Theories, volume 44 of Lecture Notes in Logic. Cambridge University Press, Cambridge (2015)

16. Tanović, P.: Generically stable regular types. J. Symb. Log. 80, 308-21 (2015)

17. Wagner, F.O.: Simple Theories, volume 503 of Mathematics and Its Applications. Kluwer Academic Publishers, Dordrecht (2000)

Publisher's Note Springer Nature remains neutral with regard to jurisdictional claims in published maps and institutional affiliations. 